\title{
Multi-scale study of pollutant transport and uptake in compacted bentonite
}

\author{
Fatiha Bouchelaghem
}

Received: / Accepted:

\begin{abstract}
In a previous work, a multi-scale model has been developed in order to investigate the impact of cation exchange and surface complexation on the hydraulic conductivity of two contrasted compacted bentonites. Hydraulic conductivity variations during the percolation of a lead nitrate solution have been simulated using occluded and connected inter-aggregate void configurations. For both clays, the results have displayed the strong connection between permeability increase and textural and structural evolutions at different scales during the process of pollutant leaching.

The present developments deal with the modelling of pollutant transport through compacted bentonite, by accounting for advective transport, molecular diffusion within the nanoscale interlayer pores and the inter-aggregate macro-voids, as well as pollutant fixation on the smectite layers' surface. The evolution of the different porous spaces is modelled on the basis of an extensive structural investigation of the solid phase conducted at the nanometer and micrometer scales. The multiscale impact of ionic exchange by heavy metal on macroscopic pollutant transport is expressed through upscaling at the different scales of organization within the compacted clay. We compare the respective contributions of hydraulic conductivity, effective diffusion and pollutant uptake to the overall pollutant transport during progressive reduction of the interlamellar space associated with clay aggregate splitting and the development of inter-aggregate pores. Lead nitrate percolation tests are simulated for a reference bentonite and a natural magnesian bentonite.
\end{abstract}

Keywords multi-scale transport model · interlamellar and inter-aggregate pores · effective diffusion tensor - effective hydraulic conductivity tensor - finite element simulation · breakthrough curves

F. Bouchelaghem

UPMC Univ Paris 06, UMR 7190, Institut Jean Le Rond d'Alembert, F-75005 Paris, France. CNRS, UMR 7190, Institut Jean Le Rond d'Alembert, F-75005 Paris, France.

Tel.: +33-144278707

Fax: +33-144278878

E-mail: fatiha.bouchelaghem@upmc.fr 


\section{Introduction}

Expansive compacted bentonites have been extensively investigated for use in the isolation of household disposal sites or in the containment of high-level radioactive waste in geological formations (Appelo et al. (2008); Baeyens and Bradbury (1997a); Boving and Grathwohl (2001); Choi and Oscarson (1996); Eriksen et al. (1999); Glaus et al. (2007); Ichikawa et al. (2004); Madsen (1998); Pusch (1996, 1999); Sato and Suzuki (2003); Sato (2005); Suzuki et al. (2004)). Recent experimental studies have been dedicated to the intrinsic role played by heavy metal retention in hydraulic conductivity variations (Jozja (2003); Jozja et al. (2003, 2006); Souli et al. (2008)), with measured saturated hydraulic conductivities ranging from $10^{-11}-10^{-12} \mathrm{~m} \cdot \mathrm{s}^{-1}$ before pollutant leaching to $10^{-10}-10^{-11} \mathrm{~m} \cdot \mathrm{s}^{-1}$ after pollutant uptake. The previous values correspond to moderate compaction states, with vertical compaction stresses around 0.2-0.5 MPa and dry densities between 0.5 and $1.5 \mathrm{~g} \cdot \mathrm{cm}^{3}$, and are comparable with hydraulic conductivity measurements performed on Montigel bentonite and Wyoming bentonite in Pusch (1996).

Compared to advection, molecular diffusion through the clay pore space is generally considered to play a dominant role in the performance of highly compacted barriers (Boving and Grathwohl (2001); Choi and Oscarson (1996); Madsen (1998); Ochs et al. (2001)), and numerous measurements of the diffusion of various cations and water tracers in water-saturated bentonites have resulted in apparent diffusion coefficients ranging from $10^{-9} \mathrm{~m}^{2} \cdot \mathrm{s}^{-1}$ to $10^{-15} \mathrm{~m}^{2} \cdot \mathrm{s}^{-1}$ depending on the compaction state, the microstructural arrangement of pore space, the diffusing species, the nature of the exchangeable cation, pore water ionic strength and temperature (Appelo et al. (2008); Choi and Oscarson (1996); Eriksen et al. (1999); Glaus et al. (2007); Kozaki et al. (1998, 2005); Madsen (1998); Molera and Eriksen (2002); Ochs et al. (2001); Sato and Suzuki (2003); Suzuki et al. (2004)).

In compacted water-saturated smectites, very thin interlayer nanopores located between the basal surfaces of stacked montmorillonite lamellae represent systematically more than half of the pore space (Choi and Oscarson (1996); Kozaki et al. (2001a); Jozja (2003)). They constitute the main domain of cation adsorption, and diffusion of species adsorbed in the interlayer takes place according to a diffuse-layer mechanism. In contrast, large micrometric pores are also encountered between clay aggregates and/or between other grains of minerals present within the clay material, where transport by diffusion and advection is unaffected by the surface.

Experimental investigations have displayed structural effects induced by heavy metal percolation through water-saturated compacted bentonites (Jozja et al. (2003); Souli et al. (2008)). Those effects appear at both the nanometer scale (reduction of particle size) and micrometer scale (micro-cracking of aggregates, fabric anisotropy), and are directly related with pore space modifications (interlamellar spacing reduction, progressive opening of macropores).

From the previous review, it appears that a realistic model of contaminant transport in compacted bentonite should be able to express that diffusion and advection take place differently in nanopores and macropores owing to surface effects, and to account for the continuous evolution of the microstructure and pore space during pollutant fixation. 
Multiphase reactive flow in ordered or disordered porous media can be addressed through the method of volume averaging presented in Quintard and Whitaker (1993). Starting from the local transport equations, weighting functions (that depend on the medium considered) are employed to obtain a generalized averaging procedure that leads to spatially smoothed transport equations and a closure problem that allows to obtain the effective transport properties on the basis of a geometrical model of the porous medium.

Among the models of reactive transport in porous media, we may mention the method of random-walk propagation of particles (Delay et al. (1993, 1996)). Mass transfer in adsorbing media is simulated by dissociating the roles of advection, dispersion, and the exchange occurring between the mobile and immobile phases. The lengths of the particles' jumps are determined first by the advective velocity and then by a random draw for each particle The fitted model parameters, such as the dispersivity or the exchange rate, have a meaning which is closer to their physical reality than that defined by macroscopic models. The model is also capable of modeling solute transport in dual-porosity media, composed of an intergranular or fracture porosity through which mobile water flows and a water-saturated matrix porosity with immobile water which can exchange solute with the mobile phase. Such models require generally a large number of particles to lead to stable solutions, and although they have been validated using analytical solutions, they may not be completely adapted to simulate practical situations of groundwater contamination or pollutant percolation experiments in the laboratory.

More specifically, during design of landfill sites, macroscopic phenomenological models (Borgesson et al. (2001); Jullien et al. (2002)) are generally employed in order to predict fluid flow and pollutant transport in the porous medium, and macroscopic Darcy's permeability is supposed to express microstructure effects on the resistance to flow. Such models are not completely satisfactory because they cannot reflect the real physical and chemical behaviour involved at the atomic and microscopic scales, which control transport phenomena in clays. Diffusive transport in compacted clays is generally described by a simple Fickian diffusion, with an empirical relationship between the effective diffusion coefficient and the diffusionaccessible porosity, or alternatively the effective diffusion coefficient is related to the solute's tracer diffusion coefficient in free water by using a correction based on a variable tortuosity coefficient (Appelo et al. (2008); Boving and Grathwohl (2001); Choi and Oscarson (1996)). In order to improve the prediction of some cation diffusion experiments, the contributions of free and sorbed cations are distinguished by the introduction of a linear sorption or distribution coefficient $K_{d}$ and a surface diffusion coefficient $D_{s}$ (Choi and Oscarson (1996); Eriksen et al. (1999); Molera and Eriksen (2002); Ochs et al. (2001)). Inter-aggregate and interlayer porosity are not modelled separately, and the presence of several adjustable parameters limits the predictive capability of this type of model.

Ichikawa et al. $(1999,2004)$ have developed a multiscale homogenization method, combining Molecular Dynamics to identify the local diffusivity and viscosity near the montmorillonite basal surfaces, and periodic homogenization to derive the homogenized diffusion and permeability coefficients for a non-sorbing solute. The effective diffusion and permeability coefficients are computed using a one-dimensional arrangement of water and lamellae which does not account for geometrical tortuosity in pore space, and does not distinguish between interlayer and external water. The computed longitudinal coefficient is then divided by three to obtain the macro- 
scopic diffusion coefficient which is assumed to be isotropic, and the resulting value is applied to simulate the transport of tritium in a buffer of high level radioactive waste.

(Baltean (1999); Baltean et al. (2003)) have employed a periodic homogenization approach with dual-scale microstructures to model the advective-diffusive transport of a passive solute through a non-evolving microstructure. The macroscopic model is obtained using the homogenization method for periodic structures with a double scale asymptotic expansion (Sanchez-Palencia (1980)). Numerical computation is performed using a finite element method for several geometries of the solid inclusions in two- and three-dimensions.

In conclusion, if we try to assess the hydro-mechanical behaviour of natural clays in presence of pollutants such as heavy metal ions, it appears that hydraulic conductivity and diffusivity evolutions are not usually connected with microfabric changes that can be derived from a multi-scale study. The bibliographical review motivates the need for further modelling of contaminant transport and uptake through compacted bentonites by accounting explicitly for their dual scale microstructure together with the evolution of the pore space distribution between nanopores and micropores.

Following a previous study on hydraulic conductivity variations during lead nitrate percolation through two compacted magnesian bentonites (Bouchelaghem and Jozja $(2009 \mathrm{a}, \mathrm{b})$ ), we propose a model of contaminant uptake and transport based on the homogenization method for locally periodic structures. As a result of the organization of free water within the pore space during pollutant leaching, three levels of description are considered during the model derivation : the microscopic level is the scale of stacked montmorillonite lamellae and the nanopore fluid whose physical properties (viscosity, pore-scale diffusion coefficient) are affected by the pore walls; the mesoscopic level relates to clay aggregates, mineral grains and inter-aggregate fluid which is not affected by the surface; the macroscopic level corresponds to the sample subjected to pollutant leaching tests in the laboratory. Advective transport, diffusion and pollutant fixation are taken into account within micropores (interlamellar and interparticle porosity), while in interaggregate macropores we consider only advection and diffusion. Ionic exchange will result in a 'multi-scale' modification of the microstructure. The homogenization model does not require any fitting parameter, the only strong assumption being the quasi-periodicity of the underlying microstructures.

The expressions for the effective diffusion tensors and the effective hydraulic tensors have been derived through upscaling at both the mesoscopic scale and the macroscopic scale. In particular, we have considered several configurations for void space organization, as the inter-aggregate macropores may develop to form a connected network or remain occluded, or may be negligible in highly compacted samples. The resulting numerical systems are solved using two-dimensional (at the mesoscopic scale) and three-dimensional (at the macroscopic scale) microstructures. Diffusion calculations have been compared with existing diffusivity measurements performed on pure smectites and natural bentonites. Finally, the transport model has been applied to a well-characterized reference montmorillonite and a natural magnesian bentonite intended for use in a landfill site. For both clays, hydraulic conductivity variations and effluent pollutant concentration have been monitored during lead nitrate percolation through an oedometer cell. 


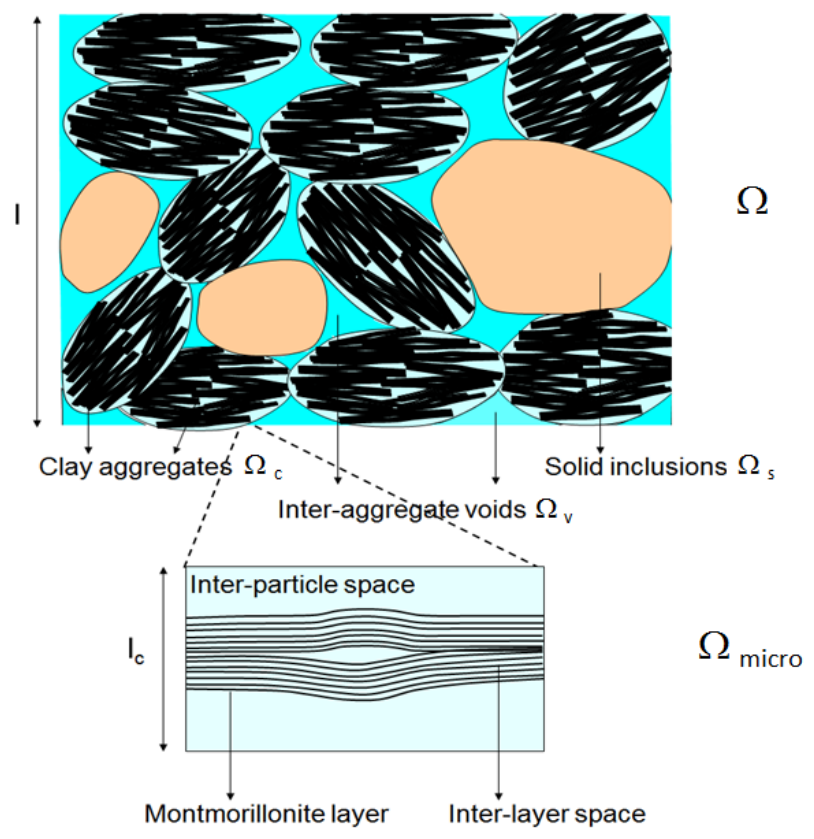

Fig. 1 Multi-scale schematic representation of bentonite: Mesoscopic cell $\Omega$ and Microscopic cell $\Omega_{\text {micro }}$

The confrontation between simulation and experimental breakthrough curves enables in particular to assess the relative contributions of diffusion and advection to pollutant transport.

\section{Microstructure description}

Based on physico-chemical investigation of bentonite samples continuously compacted in a dry state prior to water saturation (Jozja (2003); Jozja et al. (2003); Kozaki et al. (1998, 2001a); Pusch (1999); Souli et al. (2008)), the schematic description of the compacted bentonite microstructure is illustrated in Fig. 1. The representative volume element (RVE) is composed of solid mineral grains $\Omega_{s}$ and inter-aggregate voids $\Omega_{v}$ (which may form a connected network or be occluded) that are assumed to be distributed with local periodicity within a connected porous matrix $\Omega_{c}$ comprising clay lamellae (stacked in a quasi-parallel way) and watersaturated nanopores.

As both nanopores and macropores evolve during pollutant fixation, the transport problem is locally periodical. We consider a dual porosity network, nanopores are separated by a distance of order $\varepsilon^{\prime} L\left(\varepsilon^{\prime}=\frac{l_{c}}{l}\right)$, and larger pores are distributed at a distance of order $\varepsilon L\left(\varepsilon=\frac{l}{L}\right)$, with $L$ the characteristic length of the macrostructure. We assume that $\varepsilon^{\prime} \ll \varepsilon \ll 1$, which expresses the condition of separation of scales. 


\section{Transport description in the nanopores}

The contaminant concentration $c$ verifies the advection-diffusion equation (Bear and Bachmat (1998)):

$$
\frac{\partial c}{\partial t}+\nabla \cdot(\mathbf{v} c)-\nabla \cdot(D \nabla c)=0 \text { in } \Omega_{c f}
$$

where $\mathbf{v}$ is the local fluid velocity vector, and $D$ designates the scalar diffusion coefficient of the pollutant in the inter-lamellar and inter-particle pore space $\Omega_{c f}$. Owing to the particular behaviour of adsorbed water molecules (Kemper et al. (1964)), $D$ is taken to vary locally according to the viscosity distribution near basal surfaces identified in Ichikawa et al. (2004) using Molecular Dynamics simulation.

Recent studies (Baeyens and Bradbury (1997a, 1999b); Kraepiel et al. (1999)) on the various sites and mechanisms that contribute to the total sorption onto smectites have clarified the separation between interlayer adsorption (dependent on ionic strength and linear, attributed to cation exchange on the permanent charge sites) and edge adsorption (essentially $\mathrm{pH}$-dependent and non linear, resulting from surface complexation reactions). Consequently, pollutant uptake on the montmorillonite basal surface $\Gamma_{s f}$ within nanopores is expressed by the following condition:

$$
\mathbf{n} \cdot(D \nabla c)=\alpha c\left\langle c_{s a t}-c\right\rangle_{+} \text {on } \Gamma_{s f}
$$

Pollutant uptake by surface complexation on the montmorillonite edge sites $\Gamma_{\text {edge }}$ is expressed using a Langmuir type equation, which has proved adequate to describe adsorption data of heavy metal on bentonites (Brigatti et al. (1995); Viraraghavan and Kapoor (1994)):

$$
\mathbf{n} \cdot(D \nabla c)=\alpha_{\text {edge }} c \frac{1}{1+\gamma c}\left\langle c_{\text {sat }}-c\right\rangle_{+} \text {on } \Gamma_{\text {edge }}
$$

where $\Gamma_{\text {edge }}$ represents the adsorption sites located on the edges of smectite minerals.

In Eqs. (2) and (3), 〈〉 designates Mackaulay brackets:

$$
\begin{array}{ll}
\left\langle c_{s a t}-c\right\rangle_{+}=c_{\text {sat }}-c, & \text { if } c_{\text {sat }}-c>0 \\
\left\langle c_{\text {sat }}-c\right\rangle_{+}=0, & \text { if } c_{\text {sat }}-c \leq 0
\end{array}
$$

$c_{\text {sat }}$ is the upper limit for the pollutant concentration value, expressing that clay retention capacity is physically limited by the amount of available sites (Baeyens and Bradbury (1997a)). The pollutant uptake rates $\alpha$ and $\alpha_{\text {edge }}$ are not constant. They depend on the amount of interlamellar and border sites and their respective capacities (Baeyens and Bradbury (1997a, 1999b)), as well as on the solute/clay ratio and compaction state since sorbates cannot access all sorption sites on compacted clays (Choi and Oscarson (1996)). In the Langmuir-type reaction boundary condition Eq. (3), $\gamma$ can be interpreted as an equilibrium constant. 


\section{Mesoscopic model}

4.1 Mesoscopic transport equation in the clay matrix

Upscaling is performed from the microscopic scale of inter-lamellar and interparticle spaces to the mesoscopic scale of clay aggregates.

Dimensionless quantities, labelled as ()$^{\prime}$, are introduced in order to obtain the orders of magnitude of the different terms in Eqs. (1), (2) and (3):

$$
\mathbf{v}^{\prime}=\frac{\mathbf{v}}{v_{0}}, c^{\prime}=\frac{c}{c_{c}}, x^{\prime}=\frac{x}{l}, t^{\prime}=\frac{t}{T_{c}}, D^{\prime}=\frac{D}{D_{c}}, \alpha^{\prime}=\frac{\alpha}{\alpha_{c}},\left(\alpha_{\text {edge }}\right)^{\prime}=\frac{\alpha_{\text {edge }}}{\left(\alpha_{\text {edge }}\right)_{c}}
$$

We take $l=10^{-5} \mathrm{~m}$ as the representative length of the mesoscale mineral grains and clay aggregates. $v_{0}$ designates the characteristic velocity for fluid flow, and from a previous investigation of fluid flow in compacted bentonite we have (Bouchelaghem and Jozja (2009a)): $v_{0}=O\left(\left(\frac{l_{c}}{l}\right)^{2}\right)=\left(\frac{10^{-8}}{l 0^{-5}}\right)^{2}=O\left(10^{-6}\right) . T_{c}=\frac{l}{v_{0}}$ represents the characteristic time. Based on diffusivity measurements in watersaturated compacted bentonites (Glaus et al. (2007); Kozaki et al. (2010); Sato $(2005))$, we take $D_{c}=O\left(10^{-11}\right)$.

Based on the previous estimations, we obtain the following order of magnitude for the intermediate Peclet number at the mesoscopic scale:

$$
P e_{l}=\frac{l v_{0}}{D_{c}}=\frac{10^{-5} 10^{-6}}{10^{-11}}=O(1)
$$

i.e. the mesoscale convection and diffusion are of the same order of magnitude, and we insert Eq. (6) and the dimensionless variables into Eq. (1) in order to obtain the dimensionless transport equation:

$$
\frac{\partial c^{\prime}}{\partial t^{\prime}}+\nabla^{\prime} \cdot\left(\mathbf{v}^{\prime} c^{\prime}\right)-\nabla^{\prime} \cdot\left(D^{\prime} \nabla^{\prime} c^{\prime}\right)=0 \text { in } \Omega_{c f}^{\prime}
$$

while Eqs. (2) and (3) result in the following boundary conditions expressing pollutant uptake by the clay matrix:

$$
\begin{gathered}
\mathbf{n} \cdot\left(D^{\prime} \nabla^{\prime} c^{\prime}\right)=\frac{l \alpha_{c}}{D_{c}} \alpha^{\prime} c^{\prime} \text { on } \Gamma_{s f}^{\prime} \\
\mathbf{n} \cdot\left(D^{\prime} \nabla^{\prime} c^{\prime}\right)=\frac{l\left(\alpha_{\text {edge }}\right)_{c}}{D_{c}}\left(\alpha_{\text {edge }}\right)^{\prime} c^{\prime} \text { on } \Gamma_{\text {edge }}^{\prime}
\end{gathered}
$$

and we assume:

$$
\frac{l \alpha_{c}}{D_{c}}=\frac{l\left(\alpha_{e d g e}\right)_{c}}{D_{c}}=O\left(\frac{l_{c}}{l}\right)=O\left(\varepsilon^{\prime}\right)
$$

In order to express the fact that the solute concentration varies rapidly on the microscale and may also vary slowly on the mesoscale, we assume that $c^{\prime}=$ $c^{\prime}\left(x^{\prime}, y^{\prime}, t^{\prime}\right)$, where $y^{\prime}=\frac{x^{\prime}}{\varepsilon^{\prime}}$ is the local dimensionless space variable in the microstructure, and $x^{\prime}$ the mesoscopic space variable. The dependency on $y^{\prime}$ is $\Omega_{c f^{-}}^{\prime}$ periodical, and the solution is written under the form of an asymptotic development (Sanchez-Palencia (1980)): 


$$
c^{\prime}\left(x^{\prime}, y^{\prime}, t^{\prime}\right)=c^{\prime(0)}\left(x^{\prime}, y^{\prime}, t^{\prime}\right)+\varepsilon^{\prime} c^{\prime(1)}\left(x^{\prime}, y^{\prime}, t^{\prime}\right)+\left(\varepsilon^{\prime}\right)^{2} c^{\prime(2)}\left(x^{\prime}, y^{\prime}, t^{\prime}\right)+\ldots
$$

where $c^{\prime(i)}$ are $\Omega_{c f^{-}}^{\prime}$-periodical functions in $y^{\prime}$. With $y^{\prime}=\frac{x^{\prime}}{\varepsilon^{\prime}}$, the gradient operator is expressed in the following way:

$$
(\nabla)_{i}=\frac{d}{d x_{i}^{\prime}}=\frac{\partial}{\partial x_{i}^{\prime}}+\frac{1}{\varepsilon^{\prime}} \frac{\partial}{\partial y_{i}^{\prime}}=\left(\nabla_{x^{\prime}}\right)_{i}+\frac{1}{\varepsilon^{\prime}}\left(\nabla_{y^{\prime}}\right)_{i}
$$

Equations. (11) and (12) are introduced into Eqs. (7), (8) and (9). By factorizing the terms corresponding to a given order $O\left(\left(\varepsilon^{\prime}\right)^{n}\right), n=-2,-1,0, \ldots$, we obtain a series of local problems at increasing orders of approximation.

From Eqs. (7), (8) and (9) taken respectively at orders $O\left(\left(\varepsilon^{\prime}\right)^{-2}\right), O\left(\left(\varepsilon^{\prime}\right)^{-1}\right)$ and $O\left(\left(\varepsilon^{\prime}\right)^{-1}\right)$, we classically obtain that $c^{\prime(0)}$ is a mesoscopic quantity which does not depend on the microscopic variable $y^{\prime}$ :

$$
c^{\prime(0)}=c^{\prime(0)}\left(x^{\prime}, t^{\prime}\right)
$$

The linear problem resulting from Eq. (7) taken at order $O\left(\left(\varepsilon^{\prime}\right)^{-1}\right)$ and Eqs. (8) and $(9)$ taken at order $O(1)$ implies the following property of variable separation for $c^{\prime}(1)$ :

$$
c^{\prime(1)}=\vec{\beta}\left(y^{\prime}\right) \cdot \nabla_{x^{\prime}} c^{\prime(0)}\left(x^{\prime}, t^{\prime}\right)+c^{\prime 1 *}\left(x^{\prime}, t^{\prime}\right)
$$

where $c^{\prime}{ }^{1 *}\left(x^{\prime}, t^{\prime}\right)$ is an arbritrary function of $x^{\prime}$ and $t^{\prime}$.

The mesoscopic transport equation is derived from Eq. (7) considered at order $O\left(\left(\varepsilon^{\prime}\right)^{0}\right)=O(1)$. The resulting transport equation is integrated over $\Omega_{c f}^{\prime}$. By accounting for the boundary conditions Eqs. (8) and (9) at order $O\left(\varepsilon^{\prime}\right)$, the $\Omega_{c}^{\prime}$-periodicity properties of the velocity and concentration terms, the divergence theorem, the incompressibility of fluid flow $\left(\nabla_{y^{\prime}} \cdot \mathbf{v}^{\prime(0)}=0\right)$, the adherence condition $\mathbf{v}^{\prime(0)} \cdot \mathbf{n}=0$ on $\Gamma_{s f}^{\prime}$, Eqs. (13) and (14), we finally obtain after several developments:

$$
\begin{array}{r}
\frac{\left|\Omega_{c f}^{\prime}\right|}{\left|\Omega_{c}^{\prime}\right|} \frac{\partial c^{\prime(0)}}{\partial t^{\prime}}+\frac{1}{\left|\Omega_{c}^{\prime}\right|}\left(\int_{\Omega_{c f}^{\prime}} \mathbf{v}^{\prime}{ }^{(0)} d V^{\prime}\right) \cdot \nabla_{x^{\prime}} c^{\prime(0)} \\
=\frac{1}{\left|\Omega_{c}^{\prime}\right|} \int_{\Omega_{c f}^{\prime}} D^{\prime}\left(\delta_{i j}+\frac{\partial \beta_{j}}{\partial y_{i}^{\prime}}\right) d V^{\prime} \frac{\partial^{2} c^{\prime(0)}}{\partial x_{i}^{\prime} \partial x_{j}^{\prime}}+\alpha^{\prime} c^{\prime 0} \frac{\left|\Gamma_{s f}^{\prime}\right|}{\left|\Omega_{c}^{\prime}\right|}+\left(\alpha_{e d g e}\right)^{\prime} c^{\prime 0} \frac{\left|\left(\Gamma_{e d g e}\right)^{\prime}\right|}{\left|\Omega_{c}^{\prime}\right|}
\end{array}
$$

Equation (15) is then rewritten using the physical variables, we account for $v_{0}=O\left(\left(\varepsilon^{\prime}\right)^{2}\right)$ (Bouchelaghem and Jozja (2009a); Sanchez-Palencia (1980)), $T_{c}=$ $\frac{l}{v_{0}}$, Eq. (10), as well as $\frac{\left|\Omega_{c f}^{\prime}\right|}{\left|\Omega_{c}^{\prime}\right|}=\frac{\left|\Omega_{c f}\right|}{\left|\Omega_{c}\right|}, \frac{\left|\Gamma_{s f}^{\prime}\right|}{\left|\Omega_{c f}^{\prime}\right|}=l_{c} \frac{\left|\Gamma_{s f}\right|}{\left|\Omega_{c f}\right|}$ and $\frac{\left|\Gamma_{e d g e}^{\prime}\right|}{\left|\Omega_{c f}^{\prime}\right|}=l_{c} \frac{\left|\Gamma_{\text {edge }}\right|}{\left|\Omega_{c f}\right|}$. After dividing by $\frac{l}{v_{0}}$ and introducing Eq. (6) into Eq. (15), we obtain the following 
equation describing the concentration evolution at the mesoscopic scale of clay aggregates (we use Einstein's summation convention over indices $i$ and $j$ ):

$$
\pi_{c} \frac{\partial \widetilde{c}}{\partial t}+\widetilde{\mathbf{v}} \cdot \nabla \widetilde{c}=D_{i j} \frac{\partial^{2} \widetilde{c}}{\partial x_{i} \partial x_{j}}+\alpha \widetilde{c}\left\langle c_{s a t}-\widetilde{c}\right\rangle \frac{\left|\Gamma_{s f}\right|}{\left|\Omega_{c}\right|}+\alpha_{e d g e} \widetilde{c}\left\langle c_{s a t}-\widetilde{c}\right\rangle \frac{\left|\Gamma_{\text {edge }}\right|}{\left|\Omega_{c}\right|}
$$

where the volume average $\tilde{f}$ of a quantity $f(f=c, \mathbf{v})$ is defined on the elementary cell $\left|\Omega_{c}\right|$ by averaging over the fluid volume $\Omega_{c f}$ :

$$
\widetilde{f}(\mathbf{x}, t)=\frac{1}{\left|\Omega_{c}\right|} \int_{\Omega_{c f}} f(\mathbf{x}, \mathbf{y}, t) d V
$$

In Eq.(16), $\pi_{c}=\frac{\left|\Omega_{c f}\right|}{\left|\Omega_{c}\right|}$ represents the clay particles' porosity, i.e. the volume of interlamellar and inter-particle pore space per combined volume of montmorillonite and pore space. $\left|\Gamma_{s f}\right|$ is the measure of the smectite interlayers' surface where cationic exchange takes place, while $\left|\Gamma_{\text {edge }}\right|$ is the measure of the smectite egdes' surface where surface complexation occurs.

$\widetilde{\mathbf{v}}$ represents the flow velocity at the mesoscopic scale of the saturated clay aggregates, while $D_{i j}$ designate the components of the mesoscopic diffusion tensor and will be detailed in Sect. 4.2.

The pollutant transport equation (16) is supplemented with the following condition of no adsorption on the mineral grains' surface:

$$
\mathbf{D} \nabla \widetilde{c} \cdot \mathbf{n}=0 \text { on } \Gamma_{c s}
$$

4.2 Mesoscopic diffusion and hydraulic conductivity tensors

The components of the mesoscopic effective diffusion tensor $D_{i j}(i, j=1,2$ in two dimensions, $i, j=1,2,3$ in three dimensions) introduced in Eq. (16) are defined as follows:

$$
D_{i j}=\frac{1}{\left|\Omega_{c}\right|} \int_{\Omega_{c f}} D\left(\delta_{i j}+\frac{\partial \beta_{j}}{\partial y_{i}}\right) d V
$$

The field variables $\beta_{i}$ ( $i=1,2$ in two dimensions, $i=1,2,3$ in three dimensions) in Eq. (19) satisfy the system inferred from Eq. (7) at order $O\left(\left(\varepsilon^{\prime}\right)^{-1}\right)$ and Eqs. (8) and (9) at order $O(1)$. As in Bouchelaghem and Jozja (2009a), we assume that the numerical periods are symmetrical with respect to their median plane, this implies that the periodicity conditions on the outer boundary of the periodic cell can be replaced by Neumann and Dirichlet conditions on the boundary of the quarter of the cell. Consequently, $\beta_{i}$ is the unique solution of the following local diffusion problem defined over the quarter of the cell of size length $L_{e}$ (cf. Sect. 6.1):

$$
\begin{array}{r}
\nabla_{y} \cdot\left(D \nabla_{y} \beta_{i}\right)=0 \text { in } \Omega_{c f} \\
\mathbf{n} \cdot \nabla_{y} \beta_{i}=-n_{i} \text { on } \Gamma_{s f} \cup \Gamma_{\text {edge }} \\
\mathbf{n} \cdot \nabla_{y} \beta_{i}=0 \text { on } y_{j}=0 \text { and } y_{j}=\frac{L_{e}}{2}, j \neq i \\
\beta_{i}=0 \text { on } y_{i}=0 \text { and } y_{i}=\frac{L_{e}}{2}
\end{array}
$$


The mesoscopic velocity field $\widetilde{\mathbf{v}}=\left(\varepsilon^{\prime}\right)^{2} \widetilde{\mathbf{v}}^{0}$, with $\mathbf{v}^{0}$ the first order approximation of fluid velocity. By applying the conventional homogenization procedure to Stokes equations written in the case of variable fluid viscosity $\mu\left(y^{\prime}\right)$, we obtain an analog of Darcy's law (Bouchelaghem and Jozja (2009a)):

$$
\begin{aligned}
\mathbf{v}^{0} & =-\mathbf{k}_{i} \frac{\partial p^{0}}{\partial x_{i}} \\
p^{1} & =-p_{i} \frac{\partial p^{0}}{\partial x_{i}}
\end{aligned}
$$

In Eq. (21), $p^{0}$ (resp. $p^{1}$ ) is the first (resp. second) order approximation of pore fluid pressure. $\mathbf{k}_{i}=\mathbf{k}_{i}\left(\mathbf{x}, \mathbf{y}^{\prime}\right)$ is the unique solution, in a weak sense, of the following local problem solved over the quarter of an elementary cell $(l=1,2)$ :

$$
\begin{array}{r}
\mu^{0} \Delta_{y} \mathbf{k}_{l}+\nabla_{y} \mu^{0} \cdot\left(\nabla_{y} \mathbf{k}_{l}+{ }^{T} \nabla_{y} \mathbf{k}_{l}\right)+\nabla_{y} p_{l}-\mathbf{e}_{i}=\mathbf{0} \text { in } \Omega_{c f} \\
\nabla_{y} \cdot \mathbf{k}_{l}=0 \text { in } \Omega_{c f} \\
\mathbf{k}_{l}=\mathbf{0} \text { on } \Gamma_{s f} \cup \Gamma_{\text {edge }} \\
p_{l}=0, \mathbf{k}_{l} \cdot \mathbf{t}=0 \text { on } y_{l}=0, \frac{L^{e}}{2} \\
\nabla_{y} p_{l} \cdot \mathbf{n}=0, \mathbf{k}_{l} \cdot \mathbf{e}_{i}=0 \text { on } y_{i}=0, \frac{L^{e}}{2}, i \neq l \\
\nabla_{y}\left(\mathbf{k}_{l}\right) \cdot \mathbf{n}=0 \text { on } y_{i}=0, \frac{L^{e}}{2}, i=1,2
\end{array}
$$

where $\mu^{0}$ designates the first order approximation of variable fluid viscosity, and $\mathbf{e}_{i}$ is the unit vector in the direction of axis $y_{i} . \mathbf{n}$ represents the unit normal vector and $\mathbf{t}$ is the unit tangent vector on the boundaries of the cell. The volume averaging $\widetilde{\mathbf{k}}_{l}$ of $\mathbf{k}_{l}$ on the elementary cell $\left|\Omega_{c}\right|$ leads then to the determination of the hydraulic conductivity at the mesoscopic scale.

\subsection{Transport description in the macropores}

The contaminant concentration $c_{v}$ within the inter-aggregate macropores verifies the following advection-diffusion equation:

$$
\frac{\partial c_{v}}{\partial t}+\nabla \cdot\left(\mathbf{v}_{v} c_{v}\right)-D_{m} \triangle c_{v}=0 \text { in } \Omega_{v}
$$

where $\mathbf{v}_{v}$ is the local fluid velocity field and $D_{m}$ is the constant diffusion coefficient in the macropores.

The concentration is assumed to be continuous on the interface $\Gamma_{c v}$ between clay aggregates and macropores:

$$
\widetilde{c}=c_{v} \text { on } \Gamma_{c v}
$$

The normal velocities are assumed to be continuous on $\Gamma_{c v}$, as a result the condition of continuity of mass flux reduces to the continuity of diffusive flux: 


$$
(\mathbf{D} \nabla \widetilde{c}) \cdot \mathbf{n}=D_{m} \nabla c_{v} \cdot \mathbf{n} \text { on } \Gamma_{c v}
$$

The latter condition is completed by the null outflow boundary condition on $\Gamma_{v s}$ :

$$
D_{m} \nabla c_{v} \cdot \mathbf{n}=0 \text { on } \Gamma_{v s}
$$

\section{Macroscopic transport model}

To obtain the macroscopic transport equation and the macroscopic diffusion and hydraulic conductivity tensors, upscaling is carried out from the mesoscopic scale to the macroscopic scale for the locally periodical medium comprising clay aggregates, macrovoids and solid inclusions, Fig. 1. Within the porous clay matrix, pollutant transport is described by the advection-diffusion equation Eq. (16).

Three situations are investigated as the saturated macropores may be occluded, may form a connected network for contaminant transport, or may be negligible.

\subsection{Case with occluded macropores}

\subsubsection{Macroscopic transport equation}

The contaminant transport by advection and diffusion within occluded macropores $\Omega_{v}$ results from what occurs in the surrounding clay matrix. This implies that convection and diffusion are of the same order of magnitude everywhere, and from available hydraulic conductivity and diffusion experiments we make the following assumption for the macroscopic Peclet number :

$$
P e_{L}=\frac{L V_{0}}{D_{m}}=O(1)
$$

with $V_{0}$ the characteristic velocity at the mesoscopic scale, and $L=O\left(10^{-2}\right)$ the characteristic macroscopic length of the sample in the laboratory. We also define the characteristic time variable $T_{c}=\frac{L}{V_{0}}$ and the characteristic diffusion coefficient $\left(D_{c}\right)^{\text {meso }}=D_{m}=O\left(10^{-9}\right)$ in both domains $\Omega_{c}$ and $\Omega_{v}$ at the mesoscale.

By using Eq. (6) and Eq. (10), we make the following assumption concerning the reaction term:

$$
\frac{L}{V_{0}}\left(\alpha_{c} \frac{\left|\Gamma_{s f}\right|}{\left|\Omega_{c}\right|}+\left(\alpha_{e d g e}\right)_{c} \frac{\left|\Gamma_{e d g e}\right|}{\left|\Omega_{c}\right|}\right)=\frac{\varepsilon^{\prime}}{\varepsilon} l \frac{\left(\left|\Gamma_{s f}\right|+\Gamma_{\text {edge }}\right)}{\left|\Omega_{c}\right|} \frac{v_{0}}{V_{0}}=O(1)
$$

which is motivated by the high values of specific surface areas measured in compacted smectites as well as the estimation of the number of sites available for cationic exchange and surface complexation.

The asymptotic analysis is performed as in Sect. 4. Using the previous characteristic values, the system of Eqs. (16), (18), (23)-(26) is put into dimensionless form: 


$$
\begin{gathered}
\pi_{c} \frac{\partial \widetilde{c}^{\prime}}{\partial t^{\prime}}+\widetilde{\mathbf{v}}^{\prime} \cdot \nabla^{\prime} \vec{c}=\frac{1}{P e_{L}} \mathbf{D}^{\prime} \cdot \nabla^{\prime}\left(\nabla^{\prime} \widetilde{c}\right)+\frac{L}{V_{0}} \frac{\left(\alpha_{c}\left|\Gamma_{s f}\right|+\left(\alpha_{\text {edge }}\right)_{c}\left|\Gamma_{\text {edge }}\right|\right)}{\left|\Omega_{c}\right|} \alpha^{\prime} \vec{c} \text { in } \Omega_{c}^{\prime} \\
\frac{\partial c_{v}^{\prime}}{\partial t^{\prime}}+\nabla^{\prime} \cdot\left(\mathbf{v}_{v}^{\prime} c_{v}^{\prime}\right)-\frac{1}{P e_{L}} D^{\prime} \triangle^{\prime} c_{v}^{\prime}=0 \text { in } \Omega_{v}^{\prime} \\
\widetilde{c}=c_{v}^{\prime} \text { on } \Gamma_{c v}^{\prime} \\
\left(\mathbf{D}^{\prime} \nabla^{\prime} \widetilde{c}\right) \cdot \mathbf{n}=\nabla^{\prime} c_{v}^{\prime} \cdot \mathbf{n} \text { on } \Gamma_{c v}^{\prime} \\
\left(\mathbf{D}^{\prime} \nabla^{\prime} \vec{c}\right) \cdot \mathbf{n}=0 \text { on } \Gamma_{c s}^{\prime} \\
\nabla^{\prime} c_{v}^{\prime} \cdot \mathbf{n}=0 \text { on } \Gamma_{v s}^{\prime}
\end{gathered}
$$

The homogenization is performed by looking for the pollutant concentration $\widetilde{c}\left(x^{\prime}, y^{\prime}, t^{\prime}\right)$ in the clay matrix and the pollutant concentration $c_{v}^{\prime}\left(x^{\prime}, y^{\prime}, t^{\prime}\right)$ in the macropores in the form of a double scale asymptotic expansion similar to Eq. (11), with $\varepsilon$ as the "small parameter" instead of $\varepsilon^{\prime} \cdot \mathbf{y}^{\prime}$ designates now the local dimensionless position vector, and $\mathrm{x}^{\prime}$ represents the macroscopic dimensionless position vector in the macroscopic cell $\Omega$. By inserting the expression (12) of the gradient operator into the dimensionless system, we obtain the local problems at the successive orders of approximation.

At the lowest order, we define the concentration $c^{\prime(0)}$ in the basic period $\Omega^{\prime}$ in the following way:

$$
c^{\prime(0)}\left(\mathbf{x}^{\prime}, \mathbf{y}^{\prime}, t^{\prime}\right)=\left\{\begin{array}{l}
\widetilde{c}^{\prime(0)} \text { if } \mathbf{y}^{\prime} \in \Omega_{c}^{\prime} \\
c_{v}^{\prime(0)} \text { if } \mathbf{y}^{\prime} \in \Omega_{v}^{\prime}
\end{array}\right.
$$

From Eqs. (29) and (30) taken at order $O\left(\varepsilon^{-2}\right)$, Eq. (31) taken at order $O\left(\varepsilon^{0}\right)=$ $O(1)$, and the boundary conditions (32), (33), (34) taken at order $O\left(\varepsilon^{-1}\right)$, it is straightforward to deduce that $c^{\prime 0}$ is a macroscopic quantity:

$$
c^{\prime(0)}=c^{\prime(0)}\left(\mathbf{x}^{\prime}, t^{\prime}\right)
$$

At the first order of approximation, we define $c^{\prime(1)}$ :

$$
c^{\prime(1)}\left(\mathbf{x}^{\prime}, \mathbf{y}^{\prime}, t^{\prime}\right)=\left\{\begin{array}{l}
\widetilde{c}^{(1)} \text { if } \mathbf{y}^{\prime} \in \Omega_{c}^{\prime} \\
c_{v}^{(1)} \text { if } \mathbf{y}^{\prime} \in \Omega_{v}^{\prime}
\end{array}\right.
$$

The second system consists in writing Eqs. (29) and (30) at order $O\left(\varepsilon^{-1}\right)$, the continuity condition (31) at order $O\left(\varepsilon^{1}\right)$, and Neumann boundary conditions (32), (33) and (34) at order $O(1)$, and by accounting for Eq. (35), we infer the following linear decomposition for $c^{\prime 1}$ :

$$
c^{\prime(1)}\left(\mathbf{x}^{\prime}, \mathbf{y}^{\prime}, t^{\prime}\right)=\psi_{k}\left(\mathbf{y}^{\prime}\right) \frac{\partial c^{\prime(0)}\left(\mathbf{x}^{\prime}, t^{\prime}\right)}{\partial x_{k}^{\prime}}+c^{\prime 1 *}\left(\mathbf{x}^{\prime}, t^{\prime}\right)
$$


where $c^{\prime 1 *}$ is an arbitrary function of $\mathbf{x}^{\prime}$ and $t^{\prime}$, and $\psi_{k}(k=1,2,3)$ is defined in Sect. 5.1.2.

The macroscopic transport equation is obtained by considering Eqs. (29) and (30) at order $O(1)$, the Dirichlet condition (31) at order $O\left(\varepsilon^{2}\right)$, and the interface flow boundary conditions Eqs. (32), (33) and (34) at order $O(\varepsilon)$. The resulting transport equations are integrated over their respective domains $\Omega_{c}^{\prime}$ and $\Omega_{v}^{\prime}$ by accounting for conditions (35) and (36), the $\Omega^{\prime}$-periodicity of the variables, the divergence theorem, the incompressibility condition $\nabla_{y^{\prime}} \cdot \widetilde{\mathbf{v}}^{\prime 0}=0$ and the adherence condition $\widetilde{\mathbf{v}}^{\prime 0} \cdot \mathbf{n}=0$ on the interface $\Gamma_{c s}^{\prime}$ with solid grains (Bouchelaghem and Jozja (2009a)). After development, we finally obtain the macroscopic transport equation expressing the evolution of the pollutant concentration $\langle c\rangle=c^{0}\left(x^{\prime}, t^{\prime}\right)$ within the compacted bentonite:

$$
\pi \frac{\partial\langle c\rangle}{\partial t}+\langle\mathbf{V}\rangle \cdot \nabla\langle c\rangle=\mathcal{D}_{i k} \frac{\partial^{2}\langle c\rangle}{\partial x_{i} \partial x_{k}}+\frac{\left(\alpha\left|\Gamma_{s f}\right|+\alpha_{\text {edge }}\left|\Gamma_{\text {edge }}\right|\right)}{|\Omega|}\langle c\rangle\left\langle c_{\text {sat }}-c\right\rangle
$$

where $\pi=\frac{\pi_{c}\left|\Omega_{c}\right|+\left|\Omega_{v}\right|}{|\Omega|}=\frac{\pi_{c}\left|\Omega_{c}\right|}{|\Omega|}+\pi_{v}$ represents the total porosity of the compacted clay, and $\pi_{v}=\frac{\left|\Omega_{v}\right|}{|\Omega|}$ is the macroporosity. $\mathcal{D}_{i k}(i, k=1,2,3)$ represent the components of the macroscopic diffusion tensor defined in Sect. 5.1.2.

The macroscopic fluid velocity $\langle\mathbf{V}\rangle$ represents the volume average of the local fluid velocities over the interparticle and aggregate voids' space:

$$
\langle\mathbf{V}\rangle=\frac{(\varepsilon)^{2}}{|\Omega|}\left(\int_{\Omega_{c}} \widetilde{\mathbf{v}}^{0} d V+\int_{\Omega_{v}} \mathbf{v}_{v}^{0} d V\right)
$$

and is obtained from the macroscopic conductivity tensor defined in Sect. 5.1.2.

\subsubsection{Macroscopic diffusion and hydraulic conductivity tensors}

The asymptotic analysis has resulted in the introduction of the macroscopic diffusion tensor $\mathcal{D}_{i k}$ during the derivation of the macroscopic transport Eq. (37):

$$
\mathcal{D}_{i k}=\frac{1}{|\Omega|} \int_{\Omega_{c f} \cup \Omega_{v}} a_{i j}\left(\delta_{j k}+\frac{\partial \psi_{k}}{\partial y_{j}}\right) d V
$$

where $a_{i j}$ represents the local diffusion tensor at the mesoscopic scale: $a_{i j}=$ $D_{i j}$ in $\Omega_{c f}, a_{i j}=D_{m} \delta_{i j}$ in $\Omega_{v}(i, j=1,2,3)$.

The $\Omega$-periodical coefficients $\psi_{k}\left(\mathbf{y}^{\prime}\right)(k=1,2,3)$ verify the local problem consisting in Eqs. (29) and (30) at order $O\left(\varepsilon^{-1}\right)$, the continuity condition (31) at order $O\left(\varepsilon^{1}\right)$, and Neumann boundary conditions (32), (33) and (34) at order $O\left(\varepsilon^{0}\right)=O(1)$. The symmetries of the RVE employed allow to replace the conditions of periodicity with Neumann and Dirichlet boundary conditions, and the following system is solved for $\psi_{k}$ over the eighth of a three-dimensional elementary cell (of side length $L_{m}$ ):

$$
\frac{\partial}{\partial y_{i}^{\prime}}\left(a_{i j} \frac{\partial \psi_{k}}{\partial y_{j}}\right)=0 \text { in } \Omega_{c} \cup \Omega_{v}
$$




$$
\begin{array}{r}
{\left[\left[n_{i} a_{i j} \frac{\partial \psi_{k}}{\partial y_{j}}\right]\right]=-\left[\left[n_{i} a_{i k}\right]\right] \text { on } \Gamma_{c v}} \\
{\left[\left[\psi_{k}\right]\right]=0 \text { on } \Gamma_{c v}} \\
n_{i} a_{i j} \frac{\partial \psi_{k}}{\partial y_{j}}=-n_{i} a_{i k} \text { on } \Gamma_{c s} \\
n_{j} \frac{\partial \psi_{k}}{\partial y_{j}}=-n_{k} \text { on } \Gamma_{v s} \\
\mathbf{n} \cdot \nabla_{y^{\prime}} \psi_{k}=0 \text { on } y_{i}=0 \text { and } y_{i}=\frac{L_{m}}{2}, i \neq k \\
\psi_{k}=0 \text { on } y_{k}=0 \text { and } y_{k}=\frac{L_{m}}{2}
\end{array}
$$

where we use the notation $[[f]]$ to designate the discontinuity of any function $f$ which takes different values $f_{\mid \partial \Omega_{c} \cap \Gamma_{c v}}$ and $f_{\mid \partial \Omega_{v} \cap \Gamma_{c f}}$ on either side of $\Gamma_{c v}$ : [[f]] $=$ $f_{\mid \partial \Omega_{c} \cap \Gamma_{c v}}-f_{\mid \partial \Omega_{v} \cap \Gamma_{c v}}$.

In the case of non connected macropores, we have derived in Bouchelaghem and Jozja (2009a) the following expression for the macroscopic velocity at the first order of approximation, that defines the effective hydraulic conductivity tensor $\mathbf{K}^{\mathrm{ncv}}$ :

$$
\begin{array}{r}
\langle\mathbf{V}\rangle=-\mathbf{K}^{\mathrm{ncv}} \nabla_{x} p^{0} \\
K_{i j}^{\mathrm{ncv}}=\frac{1}{|\Omega|} \int_{\Omega_{c}} K_{k l} \frac{\partial}{y_{k}}\left(y_{i}+\Pi_{i}\right) \frac{\partial}{y_{l}}\left(y_{j}+\Pi_{j}\right) d V
\end{array}
$$

The following local problem is solved for $\Pi_{k}(k=1,2,3)$ on the eighth of the elementary cell :

$$
\begin{array}{r}
\frac{\partial}{\partial y_{i}}\left(K_{i j} \frac{\partial \Pi_{k}}{\partial y_{j}}\right)=-\frac{\partial K_{i k}}{\partial y_{i}} \text { in } \Omega_{c} \\
\Pi_{k}=-y_{k} \text { on } \Gamma_{c v} \\
K_{i j} \frac{\partial \Pi_{k}}{\partial y_{j}} n_{i}=-K_{i k} n_{i} \text { on } \Gamma_{c s} \\
\Pi_{k}=0 \text { on } y_{k}=0, \frac{L_{m}}{2} \\
\frac{\partial \Pi_{k}}{\partial y_{j}} n_{j}=0 \text { on } y_{i}=0, \frac{L_{m}}{2}, i \neq k
\end{array}
$$

Knowing $\Pi_{k}$, we deduce the hydraulic conductivity tensor $\mathbf{K}^{\mathrm{ncv}}$ from a simple volume averaging procedure using Eq. (41).

\subsection{Connected macropores}

In this situation the macropores $\Omega_{v}$ form a connected network for pollutant transport, and the difference with the occluded case originates from the fact that solute transport in the macropores is no longer a consequence of transport within the porous clay matrix. As observed in Bouchelaghem and Jozja (2009a), fluid flow is much slower within the clay matrix than within the porous cavities. We still verify 
$P e_{L}=O(1)$ for the macroscopic Peclet number, which implies at the macroscopic scale that convection within $\Omega_{v}$ and diffusion within $\Omega_{v}$ and $\Omega_{c}$ are of the same order of magnitude. This entails that advection can be neglected, and contaminant transport takes place by diffusion alone within the porous clay matrix.

The equations written for the transport problem at the mesoscopic scale are unchanged, except that there is no convection term in the mesoscopic pollutant transport equation Eq. (16). Consequently, the macroscopic transport equation is given by Eq. (37), in which the convective term contains only the contribution from the macropores $\left(\widetilde{\mathbf{v}}^{0}=\mathbf{0}\right)$.

Equation (39) is still valid for the macroscopic diffusion tensor. The velocity field is obtained by setting $\Omega_{c}=0$ in Eq. (38), while the local system of equations to solve on $\Omega_{v}$ is similar to the local system of Stokes equations (22) in the particular case of constant viscosity (Bouchelaghem and Jozja (2009a)):

$$
\mathbf{v}_{v}^{0}=-\mathbf{k}_{j} \frac{\partial p^{0}}{\partial x_{j}}
$$

The vector $\mathbf{k}_{j}(j=1,2,3)$, which is $\Omega_{v}$-periodical, constitutes the unique solution in $\Omega_{v}$ of the analog of the local problem of Eqs. (22) written in the particular case of constant local viscosity $\mu$ (which implies $\nabla_{y} \mu=0$ in Eq. (22)a):

$$
\begin{array}{r}
\mu \Delta_{y} \mathbf{k}_{j}+\nabla_{y} p_{j}-\mathbf{e}_{i}=\mathbf{0} \text { in } \Omega_{v} \\
\nabla_{y} \cdot \mathbf{k}_{j}=0 \text { in } \Omega_{v} \\
\mathbf{k}_{j}=\mathbf{0} \text { on } \Gamma_{v s}^{\prime}
\end{array}
$$

$\mathbf{k}_{j}, p_{j}$ being $\Omega_{v}$-periodical. $\mathbf{e}_{i}$ is the unit vector in the direction of axis $y_{i}$. By averaging $\mathbf{k}_{j}$ over $\Omega_{v}$, we obtain the macroscopic velocity field in Eq. (38) which defines the macroscopic hydraulic conductivity tensor $\mathbf{K}^{\mathrm{cV}}$ for connected macropores:

$$
\begin{array}{r}
\langle\mathbf{V}\rangle=-\mathbf{K}^{\mathrm{cv}} \nabla_{x} p^{0} \\
K_{i j}^{\mathrm{cv}}=\frac{(\varepsilon)^{2}}{|\Omega|} \int_{\Omega_{v}}\left(k_{i}\right)_{j} d V
\end{array}
$$

\subsection{No macropores}

Finally, in highly compacted bentonite the pore space is limited to interlayer porosity (Bourg et al. (2006); Kozaki et al. (2001a)). In that case, the problem reduces simply to the system of Eqs. (29) and (33). The developments are simplified since only the porous clay matrix has to be considered. Consequently, the macroscopic transport equation is given by Eq. (37), where the velocity field and the effective diffusion tensor are obtained respectively by setting $\Omega_{v}=0$ in Eqs. (38) and (39), while the volume averaging process is limited to the clay matrix volume $\Omega_{c}$. 


\section{Diffusion calculations and confrontation with existing measurements}

Finite element computation has been made for the effective diffusion tensors at the mesoscopic and macroscopic scales by solving the local problems on a variety of microstructures using Comsol Multiphysics 3.5a (Comsol (2009)). Confrontation with available measurements allows to assess the underlying model assumptions as well as the representativity of the numerical microstructures employed.

6.1 Mesoscopic diffusion calculations in the clay matrix

\subsubsection{Microstructures employed. Application to two contrasted bentonites}

The transport model has been applied to two different bentonites and percolation test simulation is presented in Sect. 7. The first bentonite (originating from Prrenjas, Albania), is a natural smectitic clay with quartz as the main impurity, the main exchangeable cations being $\mathrm{Mg}^{2+}$ and $\mathrm{Ca}^{2+}$. Prrenjas bentonite displays a poorly-organized texture characterized by a compact assembling of short and rigid particles composed of a limited number (14 in average) of smectite layers of small lateral extension. The second bentonite investigated, Wyoming bentonite, is a well-characterized montmorillonite which serves as a reference material. In Wyoming bentonite, bentonite particles contain a large number (30 in average after Mg-exchange) of long smectite lamellae of important lateral extension, stacked in a nearly-parallel way (Bouchelaghem and Jozja (2009b)). The first kind of elementary cell employed is illustrated in Fig. 2 for Prrenjas bentonite. It consists of 14 smectite lamellae stacked together and separated by interlayer water, particles being separated by inter-particle water. A similar arrangement has been retained by Ichikawa et al. (1999) and Bouchelaghem and Jozja (2009a) for hydraulic conducitivity computations, and by Ichikawa et al. (1999) for effective diffusion computations. The local system of Eqs. (21) is solved on the two-dimensional microscopic cell, and the mesoscopic diffusion tensor is obtained by inserting the components $\beta_{i}$ into Eq. (19) and averaging over $\Omega_{c f}$.

The kind of cell represented in Fig. 2 allows only the determination of the vertical component $D_{y y}$ of the mesoscopic diffusion tensor, since $n_{x}=0$ on $\Gamma_{c f}$ implies $\beta_{x}=0$ during the resolution of Eqs. (21), and $D_{x x}=\pi_{c} D_{w}$ according to Eq. (19) ( $D_{w}$ designates here the diffusion coefficient in bulk water). For the natural bentonite $\left(\pi_{c}=72.8 \%\right)$, we obtain $D_{y y}=0.707 D_{w}$, while for the reference Wyoming bentonite $\left(\pi_{c}=66.6 \%\right)$ we obtain $D_{y y}=0.611 D_{w}$. The $D_{y y}$ values computed for both bentonites are very close to $\pi_{c} D_{w}$ since in the numerical microstructures diffusion takes place essentially in the inter-particle space where it is unaffected by the viscosity variations. In contrast, measurements of the relative diffusivities along the direction parallel to the nanopore walls range between $20 \%$ and $70 \%$ of the diffusion coefficient in bulk water $D_{w}$, depending on the number of monolayers of water molecules in the interlamellar space (Kemper et al. (1964)). Consequently, in order to investigate the diffusion tensor anisotropy, we have looked for a microstructure more representative of the local tortuosity of diffusive path. Figure 3 represents a quarter of the two-dimensional spatially periodic cell. Similar square cells have been employed by Smith et al. (2004) in order to model diffusive transport through platy-clay soils, they allow to express 
Surface: diffusion_cd2

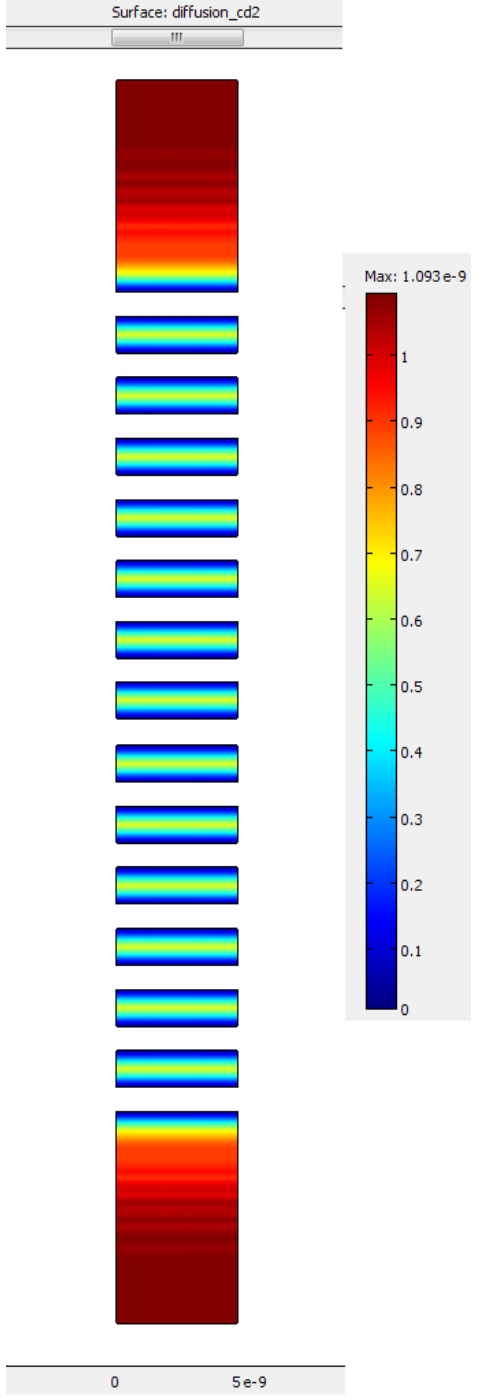

Fig. 2 Microscopic cell $\Omega_{\text {micro }}$ and variable diffusion coefficient employed for mesoscopic diffusion and hydraulic conductivity computations.

the tortuosity of diffusion path through a compacted clay at a reasonable computational cost. (Sato and Suzuki (2003); Ichikawa et al. (2004)) have also proposed a conceptual representation of bentonite with high smectite content similar to the numerical microstructures used in our study.

The clay platelets are distributed using a random generation procedure so that there is no preferred position of the clay plates, while the pseudo-random orientation $\theta$ with respect to the longitudinal axis varies between -20 and +20 , and the slenderness ratio $e$ of length to thickness is equal to 5 for Prrenjas clay 


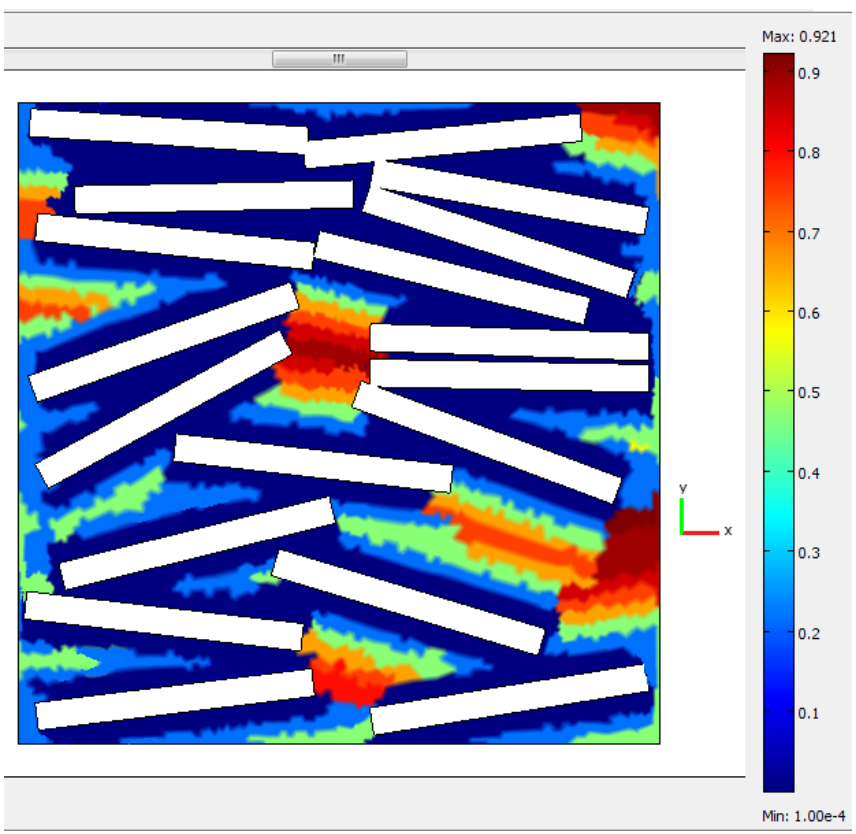

Fig. 3 One quarter of two-dimensional microscopic periodic cell $\Omega_{\text {micro }}$ employed for mesoscopic diffusion calculations. $\pi_{c}=66.7 \%$ and $e=10$. Isovalues of $\beta_{1}$ field.

and 10 for Wyoming bentonite, based on observations of compacted bentonites presented in Jozja et al. (2003, 2006).

For Prrenjas clay, we obtain $D_{x x}=0.424 D_{w}$ and $D_{y y}=0.286 D_{w}$. For Wyoming bentonite $D_{x x}=0.323 D_{w}$ and $D_{y y}=0.103 D_{w}$. The rectangular shape of the platelets favors a quasi-rectilinear diffusive path for solutes along the horizontal direction, while the tortuosity of diffusion path increases along the transverse direction as porosity decreases owing to mechanical compaction, and this effect is enhanced with increasing slenderness ratio.

\subsubsection{Comparison with experimental diffusivities}

Numerical computations of the mesoscopic diffusion tensor of a highly compacted bentonite are compared to through-diffusion measurements of ${ }^{22} \mathrm{Na}$ in a purified Na-montmorillonite from Milos (Greece) presented in Glaus et al. (2007). Owing to the high degrees of compaction, Glaus et al. (2007) assume that diffusion takes only place in the interlayer space.

Sato and Suzuki (2003) have performed diffusion measurements by varying the diffusion direction, which may coincide with the compaction direction, or be perpendicular to it, and Scanning Electron Microscopy (SEM) observations were performed in both directions. A purified bentonite of smectite content > 99 wt. $\%$ (Kunipia) has been employed under two compaction states. Each numerical value compared in Table 1 to the experimental values is an average value taken over more than 25 realizations of the microstructure. The axial values correspond to 
Table 1 Comparison between mesoscopic diffusivity coefficients measured by Sato and Suzuki (2003) and Glaus et al. (2007) and computed for a purified bentonite (pure smectite).

\begin{tabular}{cccc}
\hline Direction of diffusion & $\begin{array}{c}\rho_{d} \\
\mathrm{~g} \cdot \mathrm{cm}^{-3}\end{array}$ & $\begin{array}{c}\text { Exp. } \\
\mathrm{m}^{2} \cdot \mathrm{s}^{-1}\end{array}$ & $\begin{array}{c}\text { Num. } \\
\mathrm{m}^{2} \cdot \mathrm{s}^{-1}\end{array}$ \\
\hline Perpendicular $D_{x x}$ & 1 & $2.7 \cdot 10^{-10}$ & $2.55 \cdot 10^{-10}$ \\
Axial $D_{y y}$ Sato and Suzuki $(2003)$ & 1 & $1.2 \cdot 10^{-10}$ & $1.13 \cdot 10^{-10}$ \\
\hline Perpendicular $D_{x x}$ & 1.5 & $1.3 \cdot 10^{-10}$ & $1.15 \cdot 10^{-10}$ \\
Axial $D_{y y}$ Sato and Suzuki $(2003)$ & 1.5 & $3.3 \cdot 10^{-11}$ & $3.36 \cdot 10^{-11}$ \\
\hline Perpendicular $D_{x x}$ & 1.95 & - & $7.56 \cdot 10^{-11}$ \\
Axial Glaus et al. $(2007) D_{y y}$ & 1.95 & $2 \cdot 10^{-11}$ & $1.86 \cdot 10^{-11}$ \\
\hline
\end{tabular}

the case where the diffusion direction in the diffusion cell coincides with the vertical mechanical compaction direction, and is therefore approximately perpendicular to the longitudinal plane of clay particles.

Measurements presented in Sato and Suzuki (2003) show that diffusivity in the direction perpendicular to the compaction direction is higher than in the axial direction, and the anisotropy increases with the compaction rate. The anisotropy of the diffusion tensor, which is attributed to the elongated shape of clay platelets and preferential orientation of clay particles in the direction perpendicular to mechanical compaction, is well reproduced by the numerical mesoscopic diffusion tensor computed from Eq. (19) using microstructures similar to Fig. 3. The model compares also well with through-diffusion measurements in the compaction direction by Glaus et al. (2007). Although no diffusion data is available in the direction parallel to clay platelets, we have reported the perpendicular diffusivity coefficient in order to show that it decreases as expected with the level of compaction, and agrees well with the observed increasing anisotropy with dry density.

To conclude on the mesoscopic diffusion calculations, the computations made for the particular bentonites investigated (Prrenjas and Wyoming) lead to vertical and horizontal diffusion components consistent with existing experimental measurements. Indeed, if we take $D_{w}=110^{-9} \mathrm{~m}^{2} \cdot \mathrm{s}^{-1}$ as in Ichikawa et al. (2004), Wyoming bentonite diffusion components are close to the values measured on the less compacted bentonite in Sato and Suzuki (2003) with a similar microporosity $\left(\pi_{c}=62.2 \%\right)$. This enables us to validate the mesoscopic diffusion model. With a reasonable number of clay particles distributed at random we are able to represent the tortuosity and anisotropy of the inter-lamellar and interparticle pore space diffusion path.

\subsection{Macroscopic diffusion calculations}

\subsubsection{Microstructures employed}

\section{Non-connected macropores}

Figure 4 presents typical three-dimensional macrostructures with non-connected elongated and flat ellipsoidal voids presenting axial symmetry. In Fig. 4, a clay saturation of $75 \%$ has been employed with a macro-porosity $\pi_{v}=5 \%$, and macrovoid eccentricity ratio $e_{m}=0.979$. These microstructures have been employed for the determination of the macroscopic diffusion tensor defined by Eqs. (39), as well as 

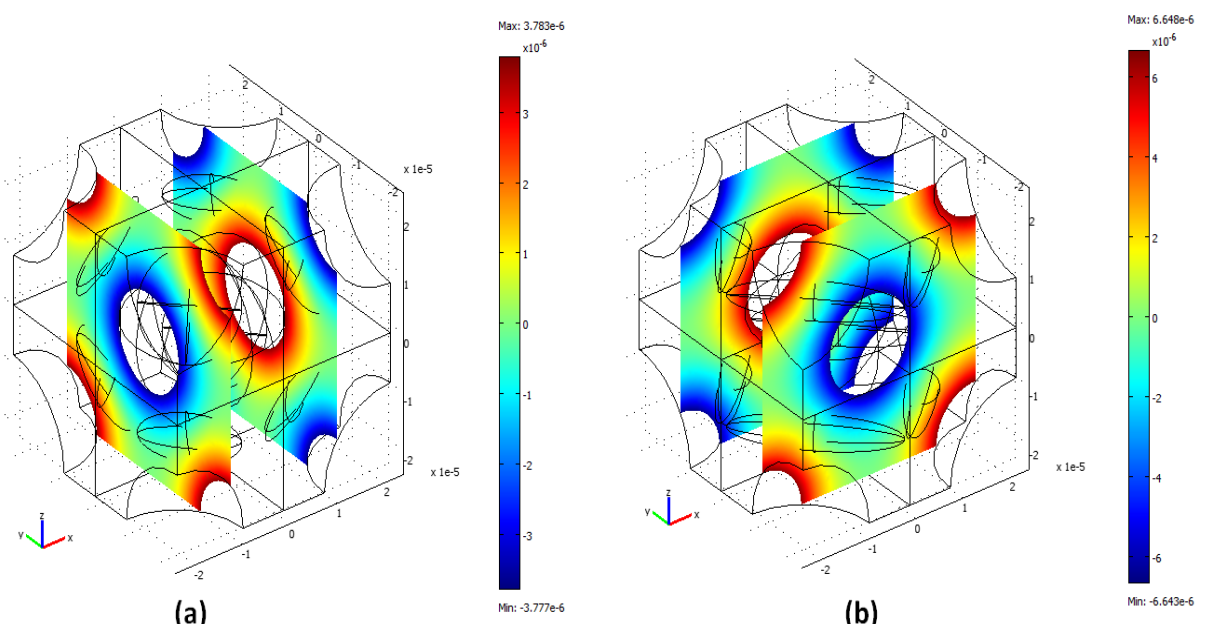

(a)

(b)

Fig. 4 Microstructures for macroscopic hydraulic conductivity and diffusion calculations. Nonconnected macrovoids. Flat ellipsoidal macrovoids and $\psi_{1}$ isovalues (a), elongated ellipsoidal macrovoids and $\psi_{2}$ isovalues (b).

in the macroscopic hydraulic conductivity computations detailed in Bouchelaghem and Jozja (2009a).

$a$ and $c$ designate respectively the largest and smallest axes lengths. In the case of elongated (resp. flat) ellipsoids, $a$ (resp. $c$ ) is the semi-length along the axis of symmetry of the spheroid, and $c$ (resp. a) denotes the semi-length along the two remaining axis of the orthonormal system of axis. The macrovoid eccentricity ratio $e_{m}=\sqrt{1-\left(\frac{c}{a}\right)^{2}}$ is taken to vary between 0 and 1 , this allows to cover a wide range of void shapes, from circular inclusions $\left(e_{m}=0\right)$ to fracture, needle-like voids $\left(e_{m}=1\right)$.

Connected macropores

In the three-dimensional microstructures, quartz and other mineral inclusions are represented by a centered cubic lattice. Three kinds of connected voids, characterized by different evolutions of the size of pore chambers and pore throats, are proposed in order to study the progressive formation of inter-connected channels at the scale of particle aggregates, easing contaminant transport by both convection and diffusion (Jozja (2003); Jozja et al. (2003); Souli et al. (2008)). The microstructures employed for macroscopic hydraulic conductivity and diffusion calculations are illustrated in Fig. 5, representing the partial derivative $\frac{\partial \psi_{k}}{\partial x_{k}}$ $(k=1,2,3)$ computed within the inter-aggregate voids and the clay matrix (clay saturation amounts to $75 \%$ ). Although idealized, such microstructures allow to compare different evolutions of the open constrictions available for nuclide diffusion and different evolutions of specific surface area, as well as to vary the threshold porosity for which macrovoids lose connectivity.

The resolution of the local system of Eqs. (41) is performed with the UMFPACK linear system solver with an average number of 10000 Lagrange quadratic finite elements and 45000 degrees of freedom, and a relative tolerance of $10^{-20}$, the only difficulty being related to the handling of the discontinuity conditions on $\Gamma_{c v}^{\prime}$. Consequently, the geometry consists of separate parts, $\Omega_{c}$ and $\Omega_{v}$, and we have to 


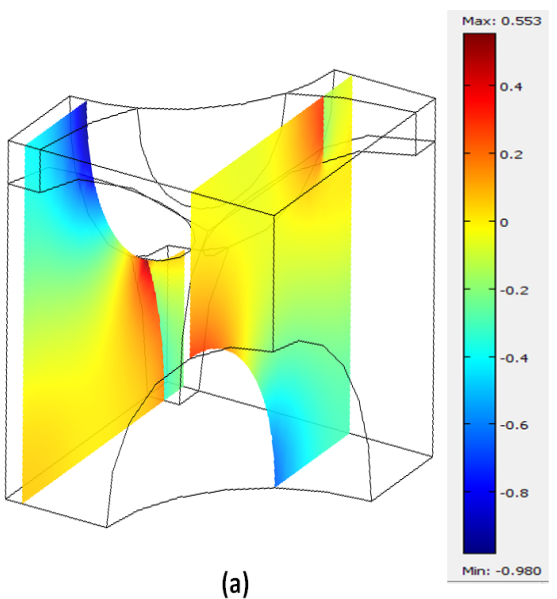

(a)
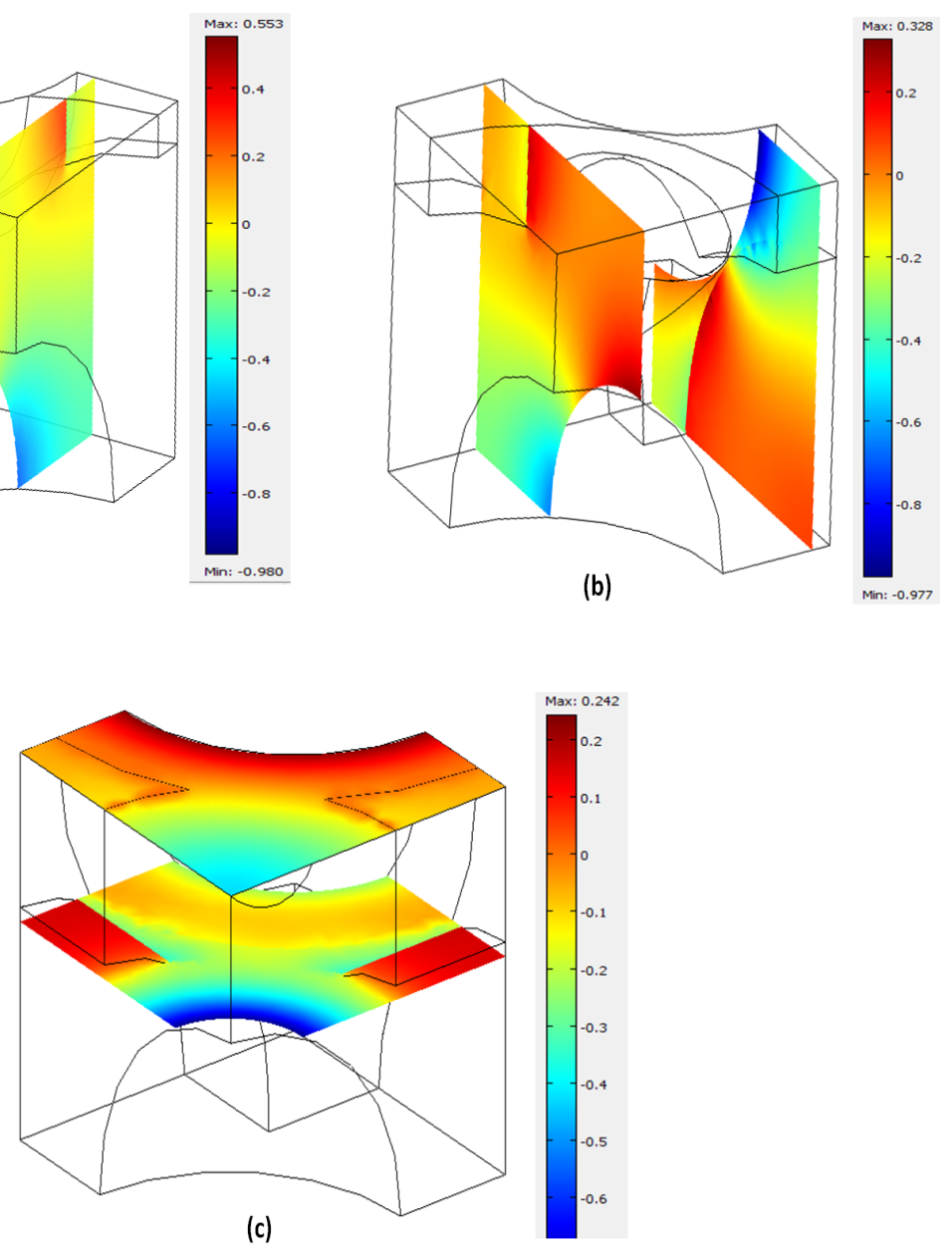

Fig. 5 One eighth of microstructures with connected macrovoids. $\pi_{v}=3.03 \%$ and $\frac{\partial \psi_{1}}{\partial x}$ isovalues (a), $\pi_{v}=8.2 \%$ and $\frac{\partial \psi_{2}}{\partial y}$ isovalues (b), $\pi_{v}=12.04 \%$ and $\frac{\partial \psi_{3}}{\partial z}$ isovalues (c).

connect the geometry, finite element mesh and equations at the interface $\Gamma_{c v}$. We use a single dependent variable $\psi_{k}(\mathrm{k}=1,2,3)$ over the entire domain $\Omega_{c} \cup \Omega_{v}, \psi_{k}$ is continuous from Dirichlet condition (41)c being imposed directly in the numerical system to solve, whereas its first derivatives $\frac{\partial \psi_{k}}{\partial y_{j}^{\prime}}$ are discontinuous according to Neumann boundary condition (41)b which is imposed in a weak form, with an ideal constraint type. As expected, $\psi_{k}(k=1,2,3)$ is continuous across $\Gamma_{c v}$ as illustrated in Fig. 4, while we observe in Fig. 5 that the flux $\nabla \psi_{k} \cdot \mathbf{n}$ is discontinuous across the interface $\Gamma_{c v}$. The numerical implementation of Eqs. (41) has been validated by confrontation with numerical and experimental macroscopic diffusivity data in two-phase media presented in Chang (1983).

\section{No macropores}




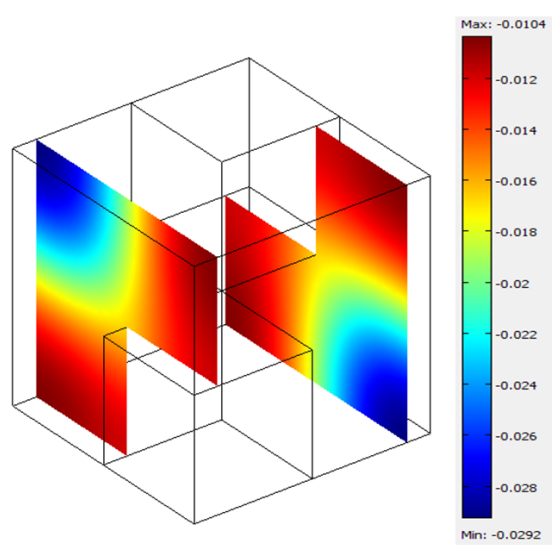

(a)

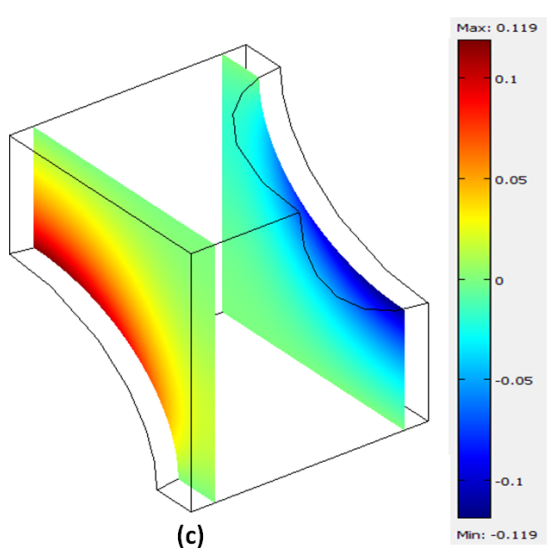

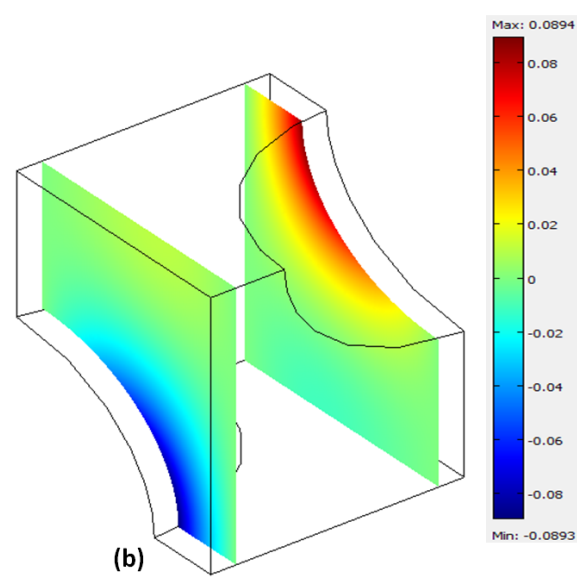

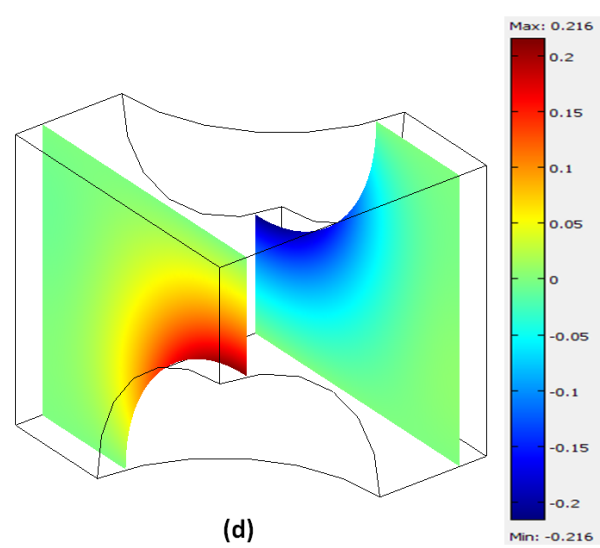

Fig. 6 One eighth of microstructures with no macro-voids. Cubical inclusions and $\psi_{1}$ isovalues (a), flat ellipsoidal inclusions $\left(e_{s}=0.745\right)$ and $\psi_{2}$ isovalues (b), elongated ellipsoidal inclusions $\left(e_{s}=0.745\right)$ and $\psi_{3}$ isovalues (c), spherical inclusions $\left(e_{s}=0\right)$ and $\psi_{3}$ isovalues (d).

When the porosity is limited to inter-layer nanopores, different geometries are adopted for the solid inclusions, in order to evaluate the effect of grain shape on the effective diffusion. As shown in Fig. 6 displaying the evolution of $\psi_{k},(k=1,2,3)$, spherical, cubical, flat ellipsoidal and elongated ellipsoidal inclusions are employed. $e_{s}$ represents the eccentricity ratio of the mineral grain, and is defined for ellipsoidal inclusions in the same way as the macrovoid eccentricity ratio $e_{m}$.

In the simulation of lead nitrate percolation through Prrenjas and Wyoming bentonites presented in Sect. 7, we consider that the grains are approximately spherical $\left(e_{s}<0.4\right)$ on the basis of experimental observations.

\subsubsection{Comparison with experimental data}

Macroscopic diffusion calculations have been compared with available data reported in Bourg et al. (2008) and Muurinen et al. (1990) on compacted watersaturated Wyoming bentonite in the direction parallel to compaction. Smectite content is estimated to be $72.5 \%$, the total porosity is $38 \%$, and the micropores 
Table 2 Comparison between computed and measured (Sato and Suzuki (2003)) macroscopic diffusivity coefficients (in $10^{-10} \mathrm{~m}^{2} \cdot \mathrm{s}^{-1}$ ).

\begin{tabular}{ccccc}
\hline $\begin{array}{c}\rho_{d} \\
\mathrm{~g} / \mathrm{cm}^{3}\end{array}$ & $\begin{array}{c}\text { Axial } D_{y y} \\
\text { Exp. }\end{array}$ & $\begin{array}{c}\text { Perp. } D_{x x} \\
\text { Exp. }\end{array}$ & $\begin{array}{c}\text { Axial } D_{y y} \\
\text { Num. }\end{array}$ & $\begin{array}{c}\text { Perp. } D_{x x} \\
\text { Num. }\end{array}$ \\
\hline 1 & 3.9 & 4.1 & 3.83 & 4.21 \\
\hline 1.5 & 2.1 & 2.6 & 2.23 & 2.54 \\
\hline
\end{tabular}

represent approximately $46 \%$ of the smectite volume. The low value of macroporosity, $\pi_{v} \simeq 5 \%$, indicates that macropores are occluded. For a dry density $\rho_{d}=$ $1.76 \mathrm{~g} \cdot \mathrm{cm}^{-3}$, the numerical diffusivity of $4.5 \cdot 10^{-11} \mathrm{~m}^{2} \cdot \mathrm{s}^{-1}$ compares well with the experimental value of $4.58 \pm 0.6 \cdot 10^{-11} \mathrm{~m}^{2} \cdot \mathrm{s}^{-1}$.

Macroscopic diffusion simulation of available diffusivity measurements (Sato and Suzuki (2003)) has also been performed in the case of connected macropores. Contrary to Kunipia, Kunigel-V1 is a natural bentonite whose smectite content is about $50 \mathrm{wt} . \%$. The pure smectite matrix occupies approximately $50 \%$ of the elementary volume. Macropores represent $29.5 \%$ of the elementary volume for $\rho_{d}=1 \mathrm{~g} \cdot \mathrm{cm}^{-3}$, and $19 \%$ for $\rho_{d}=1.5 \mathrm{~g} \cdot \mathrm{cm}^{-3}$, and we assume that they form a connected network. The confrontation with the experimental diffusivities of Sato and Suzuki (2003) is summarized in Table 2.

The computed values are fairly close to the experimental ones, and agree with SEM observations showing that contrary to a purified bentonite, there is no noticeable diffusivity anisotropy in a compacted bentonite containing a significant proportion of impurities (Sato and Suzuki (2003)).

\section{Transport simulation for two contrasted bentonites}

\subsection{Description of the percolation tests}

Percolation tests through compacted purified bentonite have been performed within an oedometer using a highly concentrated solution of lead nitrate (Jozja (2003); Jozja et al. (2003)). The first step consists in consolidating the air-dried clay sample under a pressure of $0.5 \mathrm{MPa}$. During the second step, bentonite is prehydrated and saturated with deionised water under a pressure of $0.3 \mathrm{MPa}$. Finally, a $10^{-2}$ $\mathrm{M} \mathrm{Pb}(\mathrm{NO} 3)_{2}$ solution is percolated under a pressure of $0.3 \mathrm{MPa}$ until total exchange of $\mathrm{Mg}$ and $\mathrm{Ca}$ by $\mathrm{Pb}$. The total porosity, the compacting pressure and the hydraulic head are constant during pollutant permeation through the sample. Output solutions from the oedometer are regularly analysed for these three elements using Atomic Absorption Spectrometry, while the hydraulic conductivity is calculated at regular time intervals from Darcy's law.

For Prrenjas bentonite, the experiment is stopped when the output lead concentration measured is equal to the input lead concentration of $20 \mathrm{mmol} \mathrm{l}^{-1}$. A significant increase in hydraulic conductivity has been measured after 500 hours, which is strongly correlated with pollutant concentration fluctuations observed on the breakthrough curve. The total duration of the experiment is about 850 hours, the final hydraulic conductivity is about 34 times higher than the initial value. For Wyoming bentonite, the input concentration of $20 \mathrm{mmol} \mathrm{l}^{-1}$ has not been reached, 
and at the end of the experiment (around 3800 hours), the hydraulic conductivity has increased by a moderate factor of 2 .

After lead saturation, Prrenjas and Wyoming samples have been removed from the oedometer without changing their compaction state, and investigated using Xrays Diffusion at Small Angles and Scanning Electron Microscopy using the technique of cryofracturing. The experimental observations in Jozja (2003) and numerical simulation in Bouchelaghem and Jozja (2009b) have displayed the strong correlation between permeability variations and structural variations at both the scale of interlamellar space and particle aggregates. Furthermore, the effect of lead uptake on texture is largely different for Prrenjas and Wyoming bentonites. For Prrenjas clay, inter-layer space reduction and clay particles splitting under constant total porosity occur concurrently with the progressive formation of connected inter-aggregate macrovoids easing solution flow. With Wyoming bentonite, few textural alterations are observed, and although the macroscopic porosity evolution is important ( $\pi_{v}=$ at the end of lead percolation), inter-layer spacing reduction is reported on occluded macroporosity alone.

\subsection{Confrontation with experimental breakthrough curves}

Lead percolation has been simulated for both clays using the macroscopic transport equation Eq. (37).

Concerning the porosity evolution, measurements of the water content and sample dimensions in the initial stage and after pollutant percolation have shown that the total porosity $\pi$ remains constant. However, an important re-arranging has been observed between the inter-lamellar and inter-particle voids on one side, and the inter-aggregate void space on the other side, which originate from textural evolutions at both scales and cannot be estimated from mass balance considerations (i.g. by writing the continuity equation for the solid phase). Consequently, the structural rearranging, which plays an essential role in the evolution of hydraulic conductivity, effective diffusion and reaction rate, is modelled by assuming a gradual decrease of the nanoporosity, which is reported on the inter-aggregate porosity during the percolation experiment. During the simulation, we have employed a series of microstructures with decreasing inter-lamellar spacing at the mesoscopic scale, and microstructures with increasing inter-aggregate pores at the macroscopic scale, that allow to update the hydraulic conductivity tensor, the diffusion tensor and the reaction rate at both scales during lead transport and uptake. Starting with an initial state with no macropores, we decrease the interlamellar spacing concurrently with macropores development. For Prrenjas clay, inter-lamellar spacing is reduced from two water layers to a variable inter-layering of two and a single water layer, with the progressive development of occluded macropores and connected macropores (the total porosity variation has been estimated to be $\Delta \pi_{v} \simeq$ $5 \%$ in Bouchelaghem and Jozja (2009b). For Wyoming bentonite, there is no significant microstructural changes, and although the macroporosity variation is more important ( $\Delta \pi_{v} \simeq 9.5 \%$ at the end of the percolation test), the macropores remain occluded (Bouchelaghem and Jozja (2009b)).

Concerning the reaction rate, we have distinguished between the ionic exchange expressed by Eq. (2) on the interlamellar sites, and the surface complexation cor- 
Table 3 Comparison between macroscopic diffusion and hydraulic conductivity.

\begin{tabular}{ccccccccc}
\hline Sample & \multicolumn{2}{c}{ Wyoming } & \multicolumn{2}{c}{ Prrenjas } & \multicolumn{2}{c}{ Wyoming } & \multicolumn{2}{c}{ Prrenjas } \\
& $D_{x x}\left(\mathrm{~m}^{2}\right.$ & $\left.\mathrm{s}^{-1}\right)$ & $D_{x x}\left(\mathrm{~m}^{2} \mathrm{~s}^{-1}\right)$ & $\mathrm{K}\left(\mathrm{m} \mathrm{s}^{-1}\right)$ & \multicolumn{2}{c}{$\mathrm{K}\left(\mathrm{m} \mathrm{s}^{-1}\right)$} \\
\hline Initial state & 3.23 & $10^{-10}$ & 4.24 & $10^{-10}$ & 1.8 & $10^{-12}$ & 2.2 & $10^{-11}$ \\
\hline After pollutant uptake & 3.87 & $10^{-10}$ & 6.26 & $10^{-10}$ & 3.1 & $10^{-12}$ & 7.26 & $10^{-10}$ \\
\hline
\end{tabular}

responding to Eq. (3) on the edge sites. The reaction rate is also taken to vary with time. For Prrenjas, the first 400 hours are characterized by cationic exchange only, and the reaction rate $\alpha$ has been estimated based on the cationic exchange capacity measured in Jozja (2003). After 400 hours, in agreement with the observed evolution of pore space (the splitting of clay aggregates leads to a corresponding increase in egde complexation sites), the surface complexation reaction term $\alpha_{\text {edge }} \Gamma_{\text {edge }}$ is also taken into account as the interlamellar sites become saturated. For Wyoming bentonite, we have also taken into account the two kinds of reaction sites, the transition time being around 1550 hours.

Figure 7 displays the measured and computed effluent lead concentration during the percolation test through Wyoming bentonite. Lead appears in the effluent solution after 1200 hours. Afterwards, we observe a regular and moderate increase in lead concentration, which is reproduced by the multiscale transport model through the progressive development of occluded macropores concurrently with a gradual saturation of the interlamellar and edge reaction sites.

Figure 8 compares the measured and simulation effluent lead concentration for Prrenjas clay. Lead appears in the effluent solution after 470 hours of percolation. The input concentration is reached after 830 hours approximately, indicating the end of lead fixation by clay. Compared with the reference Wyoming bentonite, the increase of effluent lead concentration is not regular, and is accompanied by noticeable fluctuations. Such fluctuations, which are not completely expressed by the model, can be attributed to the modifications of the pore space organization at several scales. The interlamellar spacing reduction and micro-aggregate splitting associated with the development of macropores affect mainly the hydraulic conductivity and the reaction rate.

7.3 Comparison between diffusion and hydraulic conductivity evolution owing to structural changes

For both Prrenjas and Wyoming clays, the macroscopic hydraulic conductivity and the macroscopic diffusion component $D_{x x}$ have been gathered in Table 3 for the initial stage (after water saturation, prior to lead nitrate percolation) and at the final stage of the experiment.

It is interesting to notice that contrary to hydraulic conductivity, effective diffusion is practically not affected by the microstructure evolution. In order to compare the relative contributions of advection and diffusion, we have performed simulations in which we set alternatively the hydraulic conductivity and the effective diffusion tensors to zero in the macroscopic transport equation, while the 


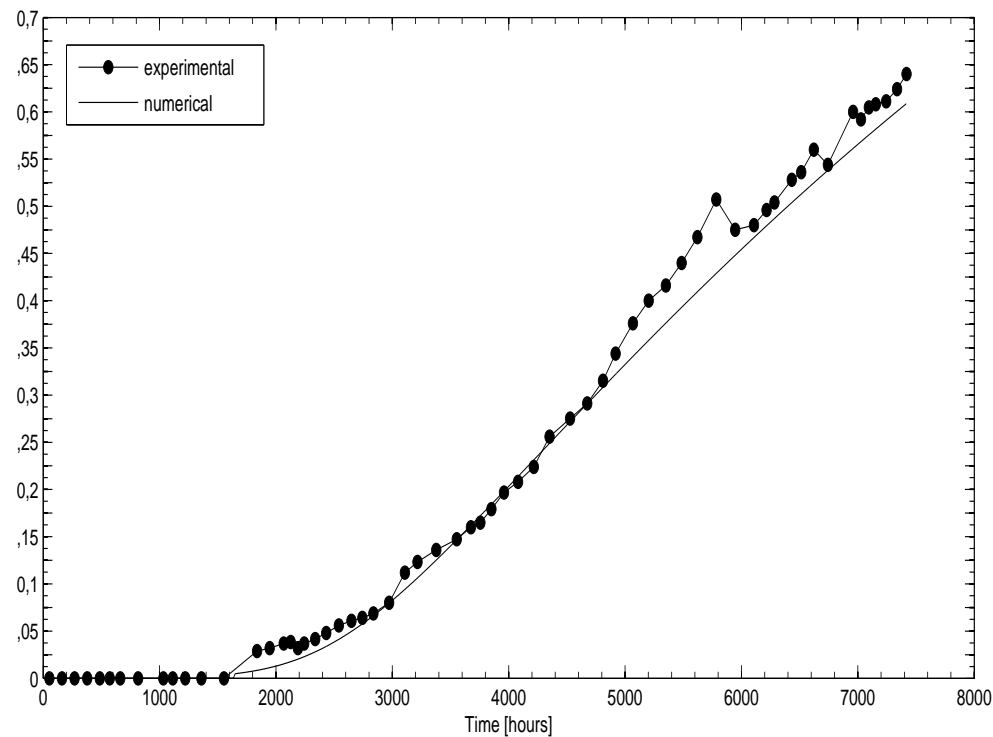

Fig. 7 Wyoming bentonite. Experimental and numerical breakthrough curves of $P b^{2+}$ during the percolation of a $10^{-2} \mathrm{M}$ solution of lead nitrate.

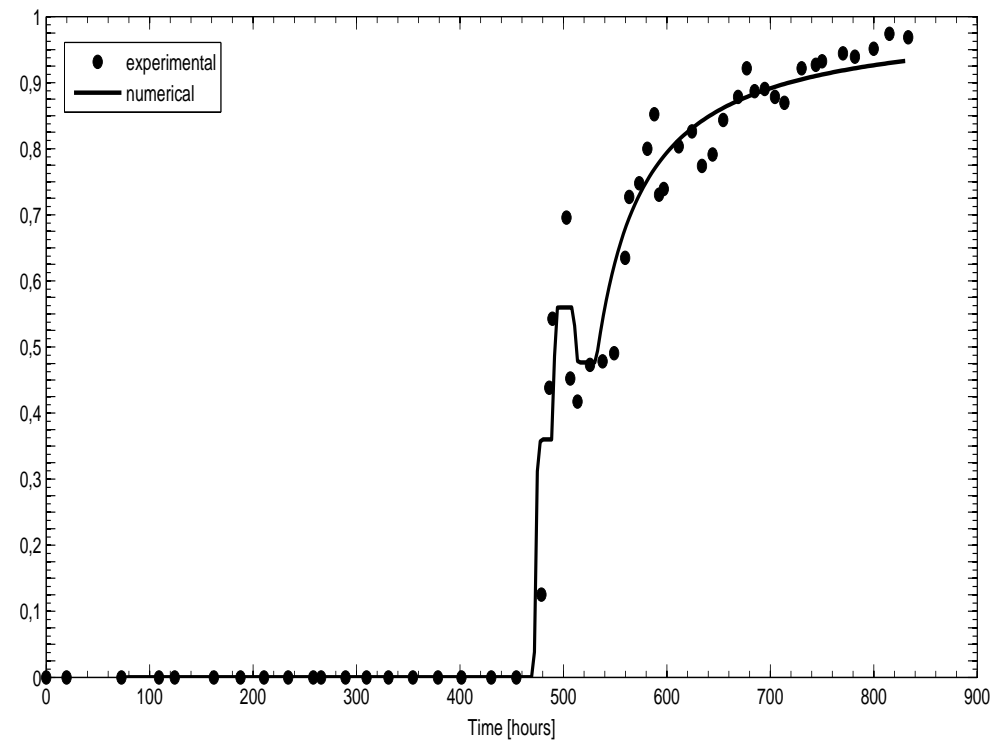

Fig. 8 Prrenjas bentonite. Experimental and numerical breakthrough curves of $P b^{2+}$ during percolation of a $10^{-2} \mathrm{M}$ solution of lead nitrate. 


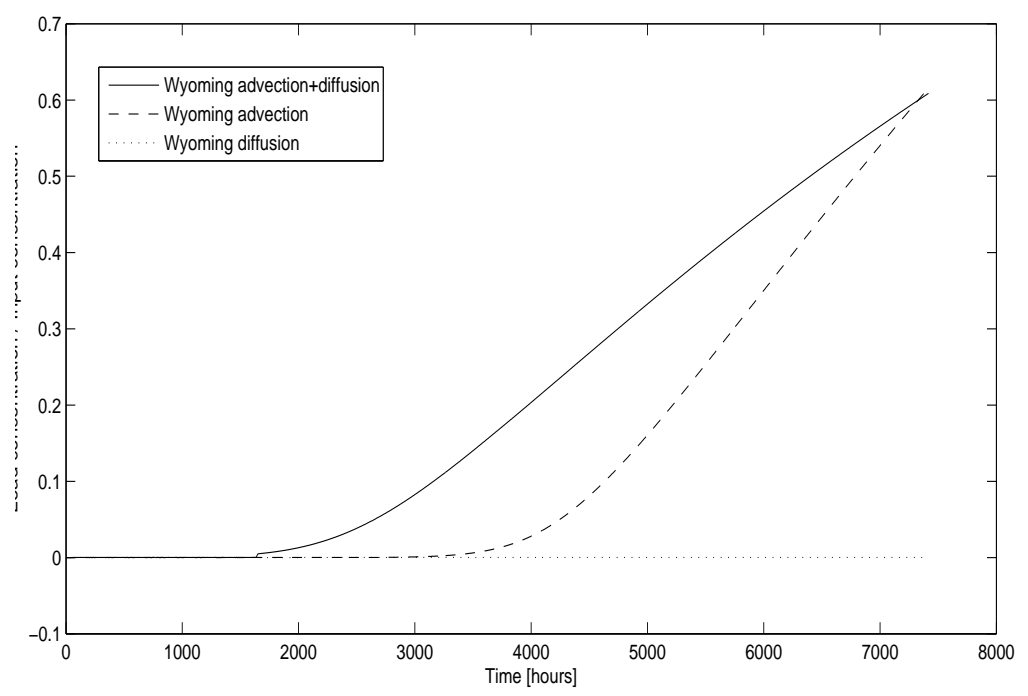

Fig. 9 Prrenjas bentonite. Comparison between the relative contributions of advection and diffusion to simulated lead effluent concentration.

reaction rate remains unchanged. The results are summarized in Fig. 9 for the reference Wyoming bentonite and in Fig. 10 for the natural Prrenjas clay. We observe that owing to structural evolutions and subsequent hydraulic conductivity increase, pollutant transport by advection plays a dominant role as compared with pollutant transport by diffusion. Due to the dependency of variable reaction rate on pollutant concentration, the total pollutant transport is not equal to the additive sum of the advective and diffusive fluxes. In particular, diffusion alone is not able to counterbalance the effect of lead uptake, while with advection alone we observe that the lead output time is notably delayed for Prrenjas clay.

\section{Conclusion}

We have presented a multi-scale model of advective-diffusive-reactive transport in a compacted water-saturated bentonite. The aim of the modelling work has been to account for the structural modifications occuring at the microscopic level of clay platelets (inter-lamellar space reduction, splitting of clay aggregates) and at the mesoscopic level of clay aggregates and mineral grains (development of occluded or connected inter-aggregate pores).

The local problems for the determination of the hydraulic conductivity and the effective diffusion tensors have been developed at both the mesoscopic scale and the macroscopic scale of the compacted bentonite sample. Owing to the progressive evolution of pore distribution during pollutant uptake, several situations have been considered. Although the micropores are always present, the macropores may be occluded or form a connected network, or may not be present in 


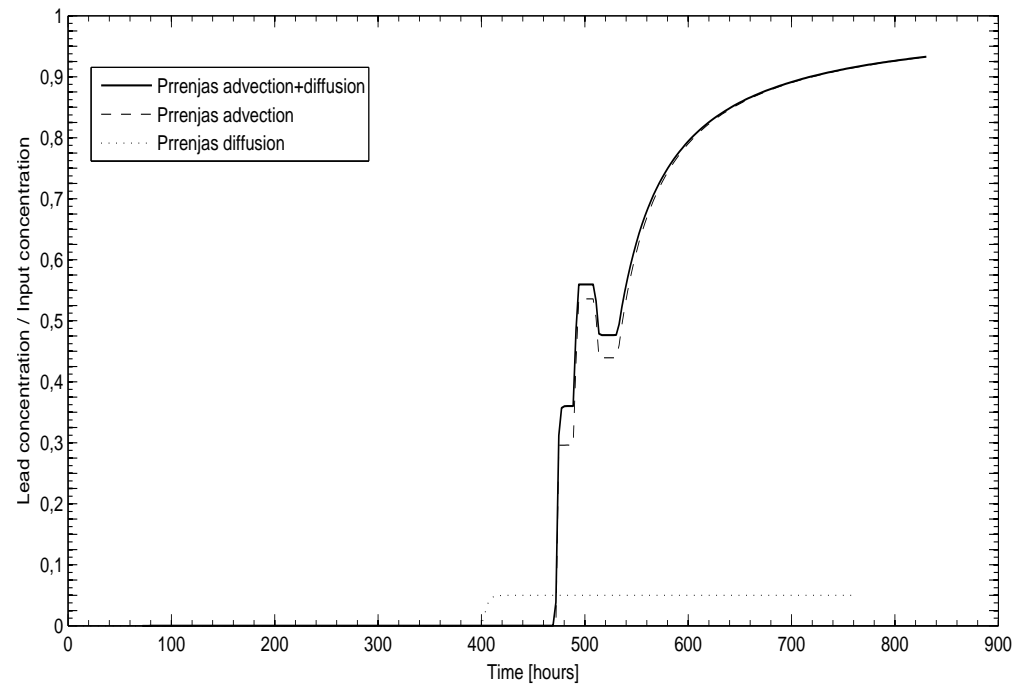

Fig. 10 Prrenjas bentonite. Comparison between the relative contributions of advection and diffusion to simulated lead effluent concentration.

highly compacted bentonite. Confrontation with diffusion and hydraulic conductivity data shows that by using simple but representative microstructures, we are able to reproduce the tortuosity and anisotropy of fluid flow and solute diffusion paths.

The model has been applied to simulate lead nitrate percolation tests through a natural bentonite characterized by a poorly organized texture, and a wellstructured reference bentonite. By relying on an thorough structural investigation of the solid phase carried out at several scales (Jozja et al. (2003, 2006); Bouchelaghem and Jozja (2009b)), we have proposed a realistic description of the bentonite texture evolution at both the nanometric and micrometric levels, which has proved able to reproduce hydraulic conductivity and lead nitrate effluent concentration measured during lead nitrate percolation.

For the natural and the reference bentonites investigated, we have compared the relative contributions of diffusive transport, advective transport and pollutant uptake to the pollutant breakthrough curves. The conclusions obtained tend to show that the usual assumption considering that advective transport of solute may be considered to be negligible in comparison to transport by molecular diffusion in compacted water-saturated bentonites may not be valid under moderate compaction states $\left(\rho_{d}<1-1.2 \mathrm{~g} \cdot \mathrm{cm}^{-3}\right)$ and important structural variations. 


\section{References}

Appelo CAJ, Vinsot A, Mettler S, Wechner S (2008) Obtaining the porewater composition of a clay rock by modeling the in- and out-diffusion of anions and cations from an in-situ experiment. J Contam Hydrol 101:67-76

Baeyens B, Bradbury MH (1997) A mechanistic description of Ni and Zn sorption on Namontmorillonite. Part I : Titration and sorption measurements. J Contam Hydrol 27:199222

Baeyens B, Bradbury MH (1997) A mechanistic description of $\mathrm{Ni}$ and Zn sorption on Namontmorillonite. Part II : modelling. J Contam Hydrol 27:223-248

Baltean D (1999) Etude de la dispersion d'un contaminant passif dans les milieux multiporeux. Doctoral dissertation, University Pierre and Marie Curie, Paris, France

Baltean D, Lévy T, Balint S (2003) Diffusion-convection in a porous medium with impervious inclusions at low flow rates. Transp Porous Media 51:19-39

Bear J, Bachmat Y (1998) Introduction to Modeling of Transport Phenomena in Porous Media. Dordrecht, The Netherlands: Kluwer Academic Publishers

Borgesson L, Chijimatsu M, Fujita T, Nguyen TS, Rutqvist J, Jing L (2001) Thermo-hydromechanical characterisation of a bentonite-based buffer material by laboratory tests and numerical back analyses. Int J Rock Mech Min Sci 38:95-104

Bouchelaghem F, Jozja N (2009) Multi-scale study of permeability evolution of a bentonite clay owing to pollutant transport. Part I. Model derivation. Eng Geol 108:119-132

Bouchelaghem F, Jozja N (2009) Multi-scale study of permeability evolution of a bentonite clay owing to pollutant transport. Part II. Application to an Mg-bentonite. Eng Geol 108:286-294

Bourg IC, Sposito G, Bourg ACM (2006) Tracer diffusion in compacted, water-saturated bentonite. Clays Clay Miner 54(3):363-374

Bourg IC, Sposito G, Bourg ACM (2008) Modeling the diffusion of $\mathrm{Na}^{+}$in compacted watersaturated Na-bentonite as a function of pore water ionic strength. Appl Geochem 23:36353641

Brigatti MF, Corradini F, Franchini GC, Mazzoni S, Medici L, Poppi L (1995) Interaction between montmorillonite and pollutants from industrial waste-waters: exchange of $\mathrm{Zn}^{2+}$ and $\mathrm{Pb}^{2+}$ from aqueous solutions. Appl Clay Sci 9:383-395

Boving TB, Grathwohl P (2001) Tracer diffusion coefficients in sedimentary rocks: correlation to porosity and hydraulic conductivity. J Contam Hydrol 53:85-100

Chang HC (1983) Effective diffusion and conduction in two-phase media : a unified approach. AIChE J 29(5):846-853

Choi JW, Oscarson DW (1996) Diffusive transport through compacted Na- and Ca-bentonite. J Contam Hydrol 22:189-202

Comsol Multiphysics 3.5a (2009) Modeling Guide, Comsol AB, Stockholm, Sweden

Delay F, Dzikowski M, de Marsily G (1993) A new algorithm for representing transport in porous media in one dimension, including convection, dispersion, and interaction with the immobile phase with first-order kinetics. Math Geol 25(6):689-711

Delay F, Rousset-Resche H, Porel G, de Marsily G (1993) Transport in a 2-D saturated porous medium: a new method for particle tracking. Math Geol 28(1):45-71

Eriksen TE, Jansson M, Molera M (1999) Sorption effects on cation diffusion in compacted bentonite. Eng Geol 54:231-236

Glaus MA, Baeyens B, Bradbury MH, Jakob A, Van Loon LR, Yaroshchuk A (2007) Diffusion of ${ }^{22} \mathrm{Na}$ and ${ }^{85} \mathrm{Sr}$ in montmorillonite: evidence of interlayer diffusion being the dominant pathway at high compaction. Environ Sci Technol 41:478-485

Ichikawa Y, Kawamura K, Nakano M, Kitayama K, Kawamura H (1999) Unified molecular dynamics and homogenization analysis for bentonite behavior: current results and future possibilities. Eng Geol 54:21-32

Ichikawa Y, Kawamura K, Fujii N, Kitayama K (2004) Microstructure and micro/macrodiffusion behavior of tritium in bentonite, Appl Clay Sci 26:75-90

Jozja N (2003) Etude de matériaux argileux albanais. Caractérisation multi-échelle d'une bentonite magnésienne. Doctoral dissertation, University of Orleans. Available online at http://tel.archives-ouvertes.fr/tel-00003740/en/

Jozja N, Baillif P, Touray JC, Pons CH, Muller F, Burgevin C (2003) Impacts multi-échelle d'un échange ( $\mathrm{Mg}, \mathrm{Ca})-\mathrm{Pb}$ et ses conséquences sur l'augmentation de la perméabilité d'une bentonite, C.R. Geosci 335:729-736 
Jozja N, Baillif P, Touray JC, Muller F, Clinard C (2006) Incidence of lead uptake on the microstructure of a (Mg,Ca)-bearing bentonite (Prrenjas, Albania). Eur J Mineral 18(3):361368

Jullien A, Proust C, Le Forestier L, Baillif P (2002) Hydro-chemio-mechanical coupling effects on permeablity and swelling behaviour of a Ca smectite soaked by $\mathrm{Cu}$ solutions. Appl Clay Sci 21:143-153

Kemper WD, Maasland DEL, Porter L (1964) Mobility of water adjacent to mineral surfaces. Soil Sci Soc Am Proc 28:164-167

Kozaki T, Fujishima A, Sato S, Ohashi H (1998) Self-diffusion of sodium ions in compacted montmorillonite. Nucl Technol 121:63-69

Kozaki T, Inada K, Sato S, Ohashi H (2001) Diffusion mechanism of chloride ions in sodium montmorillonite. J Contam Hydrol 47:159-170

Kozaki T, Fujishima A, Saito N, Sato S, Ohashi H (2005) Effects of dry density and exchangeable cations on the diffusion process of sodium ions in compacted montmorillonite. Eng Geol 81:246-254

Kozaki T, Sawaguchi T, Fujishima A, Sato S (2010) Effect of exchangeable cations on apparent diffusion of $\mathrm{Ca}^{2+}$ ions in Na- and Ca-montmorillonite mixtures. Phys Chem Earth 35:254258

Kraepiel AML, Keller K, Morel FMM (1999) A model for metal adsorption on montmorillonite. J Colloid Interface Sci 210, 43-54

Madsen FT (1998) Clay mineralogical investigations related to nuclear waste disposal. Clay Minerals 33:109-129.

Molera M, Eriksen T (2002) Diffusion of ${ }^{22} \mathrm{Na}^{+},{ }^{85} \mathrm{Sr}^{2+},{ }^{134} \mathrm{Cs}^{+}$and ${ }^{57} \mathrm{Co}^{2+}$ in bentonite clay compacted to different densities: experiments and modeling. Radiochim Acta 90:753-760

Muurinen A, Olin M, Uusheimo K (1990) Diffusion of sodium and copper in compacted sodium bentonite at room temperature. In: Proc. thirteen symposium on scientific basis for nuclear waste management. Pittsburgh: Materials Research Society, 641-647

Muurinen A, Karnland O, Lehikoinen J (2004) Ion concentration caused by an external solution into the porewater of compacted bentonite. Phys Chem Earth 29:119-127

Nakaoka K, Yamamoto S, Hasegawa H, Kawamura H, Kitayama K, Saito N, Ichikawa Y, Kawamura K, Nakano M (2004) Long-term consolidation mechanisms based on micromacro behavior and in situ XRD measurement of basal spacing of clay minerals. Appl Clay Sci 26:521-533

Neaman A, Pelletier M, Villieras F (2003) The effect of exchanged cation, compression, heating and hydration on textural properties of bulk bentonite and its corresponding purified montmorillonite. Appl Clay Sci 22:153-168

Ochs M, Lothenbach B, Wanner H, Sato H, Yui M (2001) An integrated sorption-diffusion model for the calculation of consistent distribution and diffusion coefficients in compacted bentonite. J Contam Hydrol 47:283-296

Perrins WTD, McKenzie DR, Mc Phedran RC (1979) Transport properties of regular arrays of cylinders. Proc R Soc London Ser A 369:207.

Pusch R (1996) Microstructural modelling of smectitic buffers and backfills. SKB Progress Report U-96-28

Pusch R (1999) Microstructural evolution of buffers. Eng Geol 54:33-41.

Quintard M, Whitaker S (1993) Transport in ordered and disordered porous media: volumeaveraged equations, closure problems, and comparison with experiment. Chem Eng Sci 48(14):2537-2564

Sanchez-Palencia E (1980) Non-Homogenous Media and Vibration Theory. Lecture Notes in Physics 127. Berlin: Springer-Verlag

Sato H, Suzuki S (2003) Fundamental study on the effect of an orientation of clay particles on diffusion pathway in compacted bentonite. Appl Clay Sci 23:51-60

Sato H (2005) Effects of the orientation of smectite particles and ionic strength on diffusion and activation enthalpies of $\mathrm{I}^{-}$and $\mathrm{C}_{\mathrm{S}}^{+}$ions in compacted smectite. Appl Clay Sci 29:267-281

Smith D, Pivonka P, Jungnickel C, Fityus S (2004) Theoretical analysis of anion exclusion and diffusive transport through platy-clay soils. Transp Porous Media 57:251-277

Souli H, Fleureau JM, Trabelsi Ayadi M, Besnard M (2008) Physicochemical analysis of permeability changes in the presence of zinc. Geoderma 145:1-7

Sposito G, Skipper NT, Sutton R, Park SH, Soper AK, Greathouse JA (1999) Surface geochemistry of the clay minerals. Proc Natl Acad Sci USA 96:3358-3364 
Suzuki S, Sato H, Ishidera T, Fujii N (2004) Study on anisotropy of effective diffusion coefficient and activation energy for deuterated water in compacted sodium bentonite. J Contam Hydrol 68:23-37

Viraraghavan T, Kapoor A (1994) Adsorption of mercury from wastewater by bentonite. Appl Clay Sci 9:31-49 